\title{
A proposal for $(0,2)$ mirrors of toric varieties
}

\author{
Wei Gu and Eric Sharpe \\ Physics Department, Robeson Hall (0435), \\ Virginia Tech, Blacksburg, VA 24061, U.S.A. \\ E-mail: weig8@vt.edu, ersharpe@vt.edu
}

Abstract: In this paper we propose $(0,2)$ mirrors for general Fano toric varieties with special tangent bundle deformations, corresponding to subsets of toric deformations. Our mirrors are of the form of (B/2-twisted) $(0,2)$ Landau-Ginzburg models, matching HoriVafa mirrors on the $(2,2)$ locus. We compare our predictions to $(0,2)$ mirrors obtained by Chen et al. for certain examples of toric varieties, and find that they match. We also briefly outline conjectures for analogous results for hypersurfaces in Fano toric varieties. Our methods utilize results from supersymmetric localization, which allows us to incidentally gain occasional further insights into GLSM-based $(2,2)$ mirror constructions. For example, we explicitly verify that closed string correlation functions of the original Atwisted GLSM match those of the mirror B-twisted Landau-Ginzburg model, as well as $(0,2)$ deformations thereof.

Keywords: Sigma Models, Supersymmetric Gauge Theory, Topological Field Theories, Supersymmetry and Duality

ARXIV EPRINT: 1707.05274 


\section{Contents}

1 Introduction 2

2 Review of $(2,2)$ Fano mirrors 3

2.1 General aspects 3

2.1.1 Basics 3

2.1.2 R charges 5

2.1.3 Twisted masses 5

2.2 Example with twisted masses 6

$2.3(2,2)$ in $(0,2)$ language 7

3 Proposal for $(0,2)$ Fano mirrors $\quad 8$

4 Correlation functions 12

$4.1(2,2)$ supersymmetric cases 13

$4.2(0,2)$ supersymmetric cases 18

5 Examples 20

$\begin{array}{lll}5.1 & \mathbb{P}^{1} \times \mathbb{P}^{1} & 20\end{array}$

5.1.1 First choice of $S \quad 21$

5.1.2 Second choice of $S \quad 23$

5.1.3 Comment on Adams-Basu-Sethi result 25

$\begin{array}{lll}5.2 & \mathbb{F}_{n} & 25\end{array}$

5.2.1 First choice of $S \quad 26$

$\begin{array}{lll}5.2 .2 & \text { Second choice of } S & 28\end{array}$

$\begin{array}{lll}5.3 & d P_{2} & 30\end{array}$

5.3.1 First choice of $S \quad 30$

5.3.2 Second choice of $S \quad 33$

6 Hypersurfaces in toric varieties $\quad 34$

6.1 General aspects 34

$\begin{array}{lll}6.2 & \text { Example } & 37\end{array}$

6.3 Brief comment on the quintic 40

$\begin{array}{lll}7 & \text { Conclusions } & 40\end{array}$

$\begin{array}{ll}\text { A Brief notes on }(2,2) \text { mirror ansatz } & 41\end{array}$ 


\section{Introduction}

Ordinary mirror symmetry has had a long history in string theory. This paper concerns a heterotic generalization of ordinary mirror symmetry, sometimes known as $(0,2)$ mirror symmetry. Whereas ordinary mirror symmetry relates, in simple cases, pairs of Calabi-Yau spaces $X_{1}, X_{2},(0,2)$ mirror symmetry relates pairs $\left(X_{1}, \mathcal{E}_{1}\right),\left(X_{2}, \mathcal{E}_{2}\right)$, where $\mathcal{E}_{i} \rightarrow X_{i}$ is a holomorphic vector bundle such that $\operatorname{ch}_{2}\left(\mathcal{E}_{i}\right)=\operatorname{ch}_{2}\left(T X_{i}\right)$.

Ordinary mirror symmetry is now well-understood, but $(0,2)$ mirror symmetry is still under development, and has been for a number of years (see e.g. [1-7]). Many basics have been worked out: there is a $(0,2)$ version [2] of the Greene-Plesser orbifold construction [8], there has been an attempt [3] to duplicate GLSM-based dualities [9, 10], and for the case that $\mathcal{E}$ is a deformation of the tangent bundle of a 'reflexively plain' Calabi-Yau hypersurface, there is a $(0,2)$ analogue [4] of Batyrev's construction [11-13]. Furthermore, there is now a $(0,2)$ analogue of quantum cohomology, known as quantum sheaf cohomology, which has been developed in e.g. [14-29]. The present state-of-the-art is that quantum sheaf cohomology has been computed for toric varieties and Grassmannians with deformations of the tangent bundle. (At present, however, a heterotic analogue of Gromov-Witten invariants [30] is not yet known.)

Ideally, one would like to understand mirrors to basic cases such as the quintic with a tangent bundle deformation. At present, not even such basic examples are understood. One strategy to construct such mirrors would be to use abelian duality $[9,10]$ to construct mirrors to toric ambient spaces, and then standard tricks to extrapolate to conjectures for mirrors to compact Calabi-Yau hypersurfaces.

Such a strategy was attempted in [3], who discovered that the methods previously applied in [10] seem to crucially require $(2,2)$ supersymmetry — or at least a $(0,2)$ extension will require new ideas. As a result, the $(0,2)$ version of abelian duality is not presently understood. Worse, unlike the case when [10] was written, until recently there were no known examples of $(0,2)$ Landau-Ginzburg mirrors to Fano spaces, not even for simple cases such as $\mathbb{P}^{1} \times \mathbb{P}^{1}$, which complicates efforts to extend abelian duality to $(0,2)$ cases.

As part of a program of better understanding $(0,2)$ mirror symmetry, one of the authors has been engaged with various collaborators in a program of constructing such LandauGinzburg mirrors for Fano spaces [6, 7], to help cut through the difficulties above. In those works, mirrors were constructed for $(0,2)$ GLSMs for products of projective spaces, toric del Pezzo surfaces, and Hirzebruch surfaces ${ }^{1}$ with (Euler-type) tangent bundle deformations. In each case, mirrors were constructed in a laborious non-systematic piecemeal fashion by guessing ansatzes and comparing chiral rings and correlation functions to determine coefficients - no systematic formulas applicable to all cases were produced.

In this paper we propose formulas for $(0,2) \mathrm{B} / 2$-twisted mirrors to $\mathrm{A} / 2$ models on toric Fano spaces (and closely related toric varieties), and present corresponding conjectures for hypersurfaces, for a special class of Euler-type tangent bundle deformations corresponding

\footnotetext{
${ }^{1}$ Most Hirzebruch surfaces are not Fano, but as discussed in [7], one expects them to flow to isolated vacua in the IR, so one expects to be able to use the same techniques to build a mirror to the GLSM, which is more properly interpreted as the mirror to a different geometric phase (the UV phase) of the GLSM.
} 
to a subset of 'toric' deformations. (To be clear, we are proposing a formula for LandauGinzburg mirrors, but we are not claiming to have a worldsheet dualization procedure along the lines of [10].)

We will check that our systematic construction successfully duplicates results (for this special class of deformations) for the examples of toric Fano surfaces described in $[6,7]$. The methods we present here will only apply to a subset of the deformations considered in $[6,7]$, but will produce mirrors systematically and quickly, unlike the methods used in $[6,7]$ to arrive at the results presented there.

Our methods will use ideas and results from supersymmetric localization [31], first applied to two-dimensional GLSMs in [32, 33].

We begin in section 2 by quickly reviewing existing results on GLSM-based mirror constructions in theories with $(2,2)$ supersymmetry. In section 3 we describe our proposal for $(0,2)$ mirrors to toric Fano varieties. In section 4 we describe formal arguments for why correlation functions match between the original A/2-twisted GLSM and the mirror B/2twisted Landau-Ginzburg model. In section 5 we describe several examples, checking that the predictions of our proposal match existing results worked out in $[6,7]$. In section 6 we describe how to formally extend these results to hypersurfaces, following the same pattern that has been followed for $(2,2)$ mirror symmetry.

Other recent work on two-dimensional $(0,2)$ theories from different directions includes e.g. [34-44].

\section{Review of $(2,2)$ Fano mirrors}

Let us quickly review the mirror ansatz for abelian $(2,2)$ GLSMs for Fano toric varieties in $[10]$.

\subsection{General aspects}

\subsubsection{Basics}

First, we consider a GLSM with gauge group $\mathrm{U}(1)^{k}$ and $N$ chiral superfields, with charges encoded in charge matrix $\left(Q_{i}^{a}\right)$.

Following [10], the mirror is a theory with $k$ superfields $\Sigma_{a}$, as many as U(1)s in the original GLSM, and $N$ twisted chiral fields $Y_{i}$, as many as chiral multiplets in the original GLSM, of periodicity $2 \pi i$, with superpotential

$$
W=\sum_{a=1}^{k} \Sigma_{a}\left(\sum_{i=1}^{N} Q_{i}^{a} Y_{i}-t_{a}\right)+\mu \sum_{i=1}^{N} \exp \left(-Y_{i}\right),
$$

where $\mu$ is a scale factor.

In the expression above, the $\Sigma_{a}$ act effectively as Lagrange multipliers, generating constraints

$$
\sum_{i=1}^{N} Q_{i}^{a} Y_{i}=t_{a}
$$


originating with the $\mathrm{D}$ terms of the original theory. We can solve these constraints formally ${ }^{2}$ by writing

$$
Y_{i}=\sum_{A=1}^{N-k} V_{i}^{A} \theta_{A}+\tilde{t}_{i}
$$

where $\theta_{A}$ are the surviving physical degrees of freedom, $\tilde{t}_{i}$ are solutions of

$$
\sum_{i=1}^{N} Q_{i}^{a} \tilde{t}_{i}=t_{a}
$$

and $V_{i}^{A}$ is a rank- $(N-k)$ matrix solving

$$
\sum_{i=1}^{N} Q_{i}^{a} V_{i}^{A}=0
$$

(The rank requirement goes hand-in-hand with the statement that there are $N-k$ independent $\theta_{A}$ 's.) The periodicity of the $Y_{i}$ 's will lead to interpretations of the space of $\theta_{A}$ 's in terms of LG orbifolds and character-valued fields, as we shall review later. Note that for $t_{i}, V_{i}^{A}$ satisfying the equation above,

$$
\sum_{i=1}^{N} Q_{i}^{a} Y_{i}=\sum_{i} Q_{i}^{a}\left(\sum_{A} V_{i}^{A} \theta_{A}+\tilde{t}_{i}\right)=t_{a},
$$

and so the $V_{i}^{A}$ encode a solution of the D-term constraints.

After integrating out the Lagrange multipliers, the superpotential can be rewritten as

$$
W=\mu \sum_{i=1}^{N}\left(e^{\tilde{t}_{i}} \prod_{A=1}^{N-k} \exp \left(-V_{i}^{A} \theta_{A}\right)\right) .
$$

In this language, the $(2,2)$ mirror map between A- and B-model operators is (partially) defined by

$$
\sum_{a=1}^{k} Q_{i}^{a} \sigma_{a} \leftrightarrow \mu \exp \left(-Y_{i}\right)=\mu e^{\tilde{t}_{i}} \prod_{A=1}^{N-k} \exp \left(-V_{i}^{A} \theta_{A}\right),
$$

which can be derived by differentiating (2.1) with respect to $Y_{i}$. (See for example [10, Section 3.2], where this is derived as the equations of motion of the mirror theory. In the next section, we will also see that this map is consistent with axial $\mathrm{R}$ symmetries.) In fact, this overdetermines the map - only a subset of the $Y_{i}$ 's will be independent variables solving the constraints (2.2). As we will see explicitly later, the redundant equations are equivalent to chiral ring relations (as must follow since they all arise as the same equations of motion in the mirror), and are also specified by the equations of motion derived from the superpotential $W$ above.

\footnotetext{
${ }^{2}$ The expressions given here are entirely formal, and there can be subtleties. For example, if the entries in $V_{i}^{A}$ are fractional, then as is well-known, the mirror may have orbifolds.
} 
In appendix A we will briefly outline a variation on the usual GLSM-based mirror derivation. Regardless of how the B-model mirror superpotential is obtained, it can be checked by comparing closed-string A model correlation functions between the mirror and the original A-twisted GLSM using supersymmetric localization. For $(2,2)$ theories, this can be done at arbitrary genus using the methods of $[45,46]$, whereas for $(0,2)$ theories, we can only apply analogous tests at genus zero. We will perform such correlation function checks later in this paper.

\subsubsection{R charges}

Let us take a moment to consider $\mathrm{R}$ charges. In the A-twisted theory, the axial R-charge is in general broken by nonperturbative effects, so that under an axial symmetry transformation, anomalies induce a shift in the theta angle ${ }^{3}$ by

$$
\theta^{a} \mapsto \theta^{a}+2 \alpha \sum_{i} Q_{i}^{a}, \quad t_{a} \mapsto t_{a}+2 i \alpha \sum_{i} Q_{i}^{a},
$$

for $\alpha$ parametrizing axial $\mathrm{R}$ symmetry rotations. The shift above can formally be described as

$$
\tilde{t}_{i} \mapsto \tilde{t}_{i}+2 i \alpha,
$$

(using the relation between $\tilde{t}_{i}$ and $t^{a}$ in (2.4)). In the same vein, under the same axial $\mathrm{R}$ symmetry, the mirror field $Y_{i}$ transforms as

$$
Y_{i} \mapsto Y_{i}+2 i \alpha,
$$

so that $\exp \left(-Y_{i}\right)$ has axial R-charge 2 . If we take $\Sigma_{a}$ to also have axial R-charge 2 , then it is easy to verify that the entire mirror superpotential (2.1) has axial R-charge 2, as desired, taking the $t$ 's to have nonzero R-charge as described. In addition, the operator mirror map (2.7) is also consistent with axial R-charges in that case.

\subsubsection{Twisted masses}

One can also consider adding twisted masses. Recall that a twisted mass can be thought of as the vev of a vector multiplet, gauging some flavor symmetry. Taking the vev removes the gauge field, gauginos, and auxiliary field, and replaces them with a single mass parameter $\tilde{m}$, corresponding to the vev of the $\sigma$ field. In the notation of [47, Eq. (2.19)], this means, for a single $\mathrm{U}(1)$ flavor symmetry that acts on a field $\phi_{i}$ with charge $Q_{F, i}$, we add terms to the action of the form

$$
-2|\tilde{m}|^{2} \sum_{i} Q_{F, i}^{2}\left|\phi_{i}\right|^{2}-\sqrt{2} \sum_{i} Q_{F, i}\left(\overline{\tilde{m}}_{+, i} \psi_{-, i}+\tilde{m} \bar{\psi}_{-, i} \psi_{+, i}\right)
$$

In the present case, for a toric variety with no superpotential, there are at least as many flavor symmetries as chiral superfields modulo gauged $\mathrm{U}(1)$ s, i.e. at least $N-k \mathrm{U}(1)$ flavor symmetries. (There can also be nonabelian components.) For simplicity, we will

\footnotetext{
${ }^{3}$ This should not be confused with the fundamental field $\theta_{A}$ defined earlier.
} 
simply allow for a twisted mass $\tilde{m}_{i}$ associated to each chiral superfield, and will not try to distinguish between those related by gauge U(1)s.

Including twisted masses $\tilde{m}_{i}$, the full mirror superpotential (before integrating out $\Sigma$ 's) takes the form

$$
W=\sum_{i=1}^{N}\left(\sum_{a=1}^{k} \Sigma_{a} Q_{i}^{a}+\tilde{m}_{i}\right)\left(Y_{i}-\tilde{t}_{i}\right)+\mu \sum_{i=1}^{N} \exp \left(-Y_{i}\right) .
$$

This expression manifestly has consistent axial R-charge 2 (using the 'modified' R-charge that acts on $\tilde{t}_{i}$ ). It differs from the more traditional expression [10, Eq. (3.86)]

$$
W=\sum_{a=1}^{k} \Sigma_{a}\left(\sum_{i} Q_{i}^{a} Y_{i}-t_{a}\right)+\sum_{i=1}^{N} \tilde{m}_{i} Y_{i}+\mu \sum_{i=1}^{N} \exp \left(-Y_{i}\right),
$$

by a constant term (proportional to $\sum_{i=1}^{N} \tilde{m}_{i} \tilde{t}_{i}$ ), and so defines the same physics.

After including twisted masses, the operator mirror map becomes

$$
\sum_{a=1}^{k} Q_{i}^{a} \sigma_{a}+\tilde{m}_{i} \leftrightarrow \mu \exp \left(-Y_{i}\right)
$$

Note that both sides of this expression are consistent with the (modified) R-charge assignments described above.

Generically in this paper we will absorb $\mu$ into a redefinition of the $Y_{i}^{\prime}$ 's, and so not write it explicitly, but we mention it here for completeness.

Finally, we should remind the reader that in addition to the superpotential above, one may also need to take an orbifold to define the theory, as is well-known. This will happen if, for example, some of the entries in $\left(V_{i}^{A}\right)$ are fractions, in order to reflect ambiguities in taking the roots implicit in resulting expressions such as $\exp \left(-V_{i}^{A} \theta_{A}\right)$.

\subsection{Example with twisted masses}

To give another perspective, in this section we will review the $(2,2)$ mirror to the GLSM for $\operatorname{Tot}\left(\mathcal{O}(-n) \rightarrow \mathbb{P}^{2}\right)$, for $n \leq 3$ (and no superpotential), and to make this interesting, we will include twisted masses $\tilde{m}_{i}$, correspnding to phase rotations of each field.

The charge matrix for this GLSM is

$$
Q=(1,1,1,-n)
$$

and following the usual procedure, the D terms constrain the dual (twisted) chiral superfields as

$$
Y_{1}+Y_{2}+Y_{3}-n Y_{p}=t .
$$

The standard procedure at this point is to eliminate $Y_{p}$, and write the dual potential in terms of $Y_{1-3}$, taking a $\mathbb{Z}_{n}$ orbifold to account for the fractional coefficients of the $Y_{i}$ and its periodicity. In other words,

$$
Y_{p}=\frac{1}{n}\left(Y_{1}+Y_{2}+Y_{3}-t\right),
$$


hence the $(2,2)$ superpotential is given by

$$
\begin{aligned}
W= & \sum_{i} \tilde{m}_{i} Y_{i}+\exp \left(-Y_{1}\right)+\exp \left(-Y_{2}\right)+\exp \left(-Y_{3}\right)+\exp \left(-Y_{p}\right), \\
= & \sum_{i} \tilde{m}_{i} Y_{i}+\left(\exp \left(-Y_{1} / n\right)\right)^{n}+\left(\exp \left(-Y_{2} / n\right)\right)^{n}+\left(\exp \left(-Y_{3} / n\right)\right)^{n} \\
& +\exp (-t / n) \exp \left(-Y_{1} / n\right) \exp \left(-Y_{2} / n\right) \exp \left(-Y_{3} / n\right) .
\end{aligned}
$$

Phrased more simply, if we define $Z_{i}=\exp \left(-Y_{i} / n\right)$, then the $(2,2)$ mirror theory is, as expected, a $\mathbb{Z}_{n}$ orbifold with superpotential

$$
W=-\sum_{i} \tilde{m}_{i} n \ln Z_{i}+Z_{1}^{n}+Z_{2}^{n}+Z_{3}^{n}+\exp (-t / n) Z_{1} Z_{2} Z_{3},
$$

with the understanding that the fundamental fields are $Y_{i}$ s not $Z_{i}$ s. (For hypersurfaces, the fundamental fields will change.)

Later, we will use the matrices $\left(V_{i}^{A}\right)$ extensively, so in that language, the change of variables above is encoded in

$$
\left(V_{i}^{A}\right)=\left[\begin{array}{llll}
1 & 0 & 0 & 1 / n \\
0 & 1 & 0 & 1 / n \\
0 & 0 & 1 & 1 / n
\end{array}\right]
$$

Then, we write $Y_{i}=V_{i}^{A} \theta_{A}$, and so

$$
Y_{1}=\theta_{1}, \quad Y_{2}=\theta_{2}, \quad Y_{3}=\theta_{3}, \quad Y_{p}=(1 / n)\left(\theta_{1}+\theta_{2}+\theta_{3}-t\right)
$$

Let us next discuss the operator mirror map. This is given by

$$
\begin{aligned}
& \exp \left(-Y_{1}\right)=Z_{1}^{n} \leftrightarrow \sigma, \\
& \exp \left(-Y_{2}\right)=Z_{2}^{n} \leftrightarrow \sigma, \\
& \exp \left(-Y_{3}\right)=Z_{3}^{n} \leftrightarrow \sigma, \\
& \exp \left(-Y_{p}\right)=Z_{1} Z_{2} Z_{3} \exp (-t / n) \leftrightarrow-n \sigma .
\end{aligned}
$$

\section{$2.3(2,2)$ in $(0,2)$ language}

Now, let us describe $(2,2)$ mirrors in $(0,2)$ language, as preparation for describing more general $(0,2)$ mirrors. Let $\left(\Sigma_{a}, \Upsilon_{a}\right)$ be the $(0,2)$ chiral and Fermi components of $\Sigma_{a}$, and $\left(Y_{i}, F_{i}\right)$ the $(0,2)$ chiral and Fermi components of $Y_{i}$. Then, the $(2,2)$ superpotential $(2.8)$ is given in $(0,2)$ superspace by

$$
W=\sum_{a=1}^{k}\left[\Upsilon_{a}\left(\sum_{i=1}^{N} Q_{i}^{a} Y_{i}-t_{a}\right)+\sum_{i=1}^{N} \Sigma_{a} Q_{i}^{a} F_{i}\right]-\mu \sum_{i=1}^{N} F_{i} \exp \left(-Y_{i}\right)+\sum_{i=1}^{N} \tilde{m}_{i} F_{i}
$$

We integrate out $\Sigma_{a}, \Upsilon_{a}$ to get the constraints

$$
\sum_{i=1}^{N} Q_{i}^{a} Y_{i}=t_{a}, \quad \sum_{i=1}^{N} Q_{i}^{a} F_{i}=0
$$


which we solve with the $V_{i}^{A}$ by writing

$$
Y_{i}=\sum_{A=1}^{N-k} V_{i}^{A} \theta_{A}+\tilde{t}_{i}, \quad F_{i}=\sum_{A=1}^{N-k} V_{i}^{A} G_{A}
$$

where $\left(\theta_{A}, G_{A}\right)$ are the chiral and Fermi components of the $(2,2)$ chiral superfields $\theta_{A}$. After integrating out the constraints, the $(0,2)$ superpotential becomes

$$
W=\sum_{i=1}^{N} \sum_{A=1}^{N-k} G_{A} V_{i}^{A}\left(\tilde{m}_{i}-\mu \exp \left(-Y_{i}\right)\right)=\sum_{i=1}^{N} \sum_{A=1}^{N-k} G_{A} V_{i}^{A}\left(\tilde{m}_{i}-\mu e^{\tilde{t}_{i}} \prod_{B=1}^{N-k} \exp \left(-V_{i}^{B} \theta_{B}\right)\right) .
$$

As is standard, we remind that reader that depending upon the entries in $\left(V_{i}^{A}\right)$, the mirror may be a LG orbifold, which are required to leave $W$ invariant.

In this language, the $(2,2)$ mirror map between A- and B-model operators is (partially) defined by

$$
\sum_{a=1}^{k} Q_{i}^{a} \sigma_{a}+\tilde{m}_{i} \leftrightarrow \mu \exp \left(-Y_{i}\right)=\mu e^{\tilde{t}_{i}} \prod_{A=1}^{N-k} \exp \left(-V_{i}^{A} \theta_{A}\right)
$$

which can be derived by differentiating (2.10) with respect to $F_{i}$.

In most of the rest of this paper, we will absorb $\mu$ into a field redefinition of the $Y_{i} \mathrm{~s}$ for simplicity, but we include it here for completeness.

\section{Proposal for $(0,2)$ Fano mirrors}

We restrict to $(0,2)$ theories obtained by (some) toric deformations of abelian $(2,2)$ GLSMs for Fano spaces, by which we mean physically that we choose $E$ 's such that $E_{i} \propto \phi_{i}$, where on the $(2,2)$ locus $\phi_{i}$ is the chiral superfield paired with the Fermi superfield whose superderivative is $E_{i}$.

In addition, to define a mirror, we also make another choice, namely we pick an invertible $^{4} k \times k$ submatrix, of the charge matrix $\left(Q_{i}^{a}\right)$, which we will denote $S$. The choice of $S$ will further constrain the allowed toric deformations - for a given $S$, we only consider some toric deformations. Our mirror will depend upon the choice of $S$, and since different $S$ 's will yield different allowed bundle deformations, there need not be a simple coordinate transformation relating results for different choices of $S$ in general. Furthermore, $S$ is only relevant for bundle deformations - it does not enter $(2,2)$ locus computations, and so it has no analogue within [10].

For a given choice of $S$, in the A/2 model, write

$$
E_{i}=\sum_{a=1}^{k} \sum_{j=1}^{N}\left(\delta_{i j}+B_{i j}\right) Q_{j}^{a} \sigma_{a} \phi_{i}
$$

\footnotetext{
${ }^{4}$ We assume that the charge matrix does indeed have an invertible $k \times k$ submatrix. If not, then the theory has at least one free decoupled $\mathrm{U}(1)$, and after performing a change of basis to explicitly decouple those U(1)'s, our analysis can proceed on the remainder.
} 
where in the expression above, we do not sum over $i$ 's. The $(0,2)$ deformations we will consider are encoded in the matrices $B_{i j}$, where $B_{i j}=0$ if $i$ defines a column of the matrix $S$. Note that, at least on its face, this does not describe all possible Euler-sequence-type $(0,2)$ deformations, but only a special subset. We will give a mirror construction for that special subset.

Then, the mirror can be described by a collection of $\mathbb{C}^{\times}$-valued fields $Y_{i}$ (just as on the $(2,2)$ locus, dual to the chiral superfields of the original theory), satisfying the same D-term constraints as on the $(2,2)$ locus, and with $(0,2)$ superpotential

$$
\begin{aligned}
W= & \sum_{a=1}^{k}\left[\Upsilon_{a}\left(\sum_{i=1}^{N} Q_{i}^{a} Y_{i}-t_{a}\right)+\sum_{i=1}^{N} \Sigma_{a} Q_{i}^{a} F_{i}\right] \\
& -\mu \sum_{i} F_{i} \exp \left(-Y_{i}\right)+\mu \sum_{i} F_{i}\left(\sum_{i_{S}, j, a} B_{i j} Q_{j}^{a}\left[\left(S^{-1}\right)^{T}\right]_{a i_{S}} \exp \left(-Y_{i_{S}}\right)\right),
\end{aligned}
$$

where $i_{S}$ denotes an index running through the columns of $S$, and where the second term was chosen so that the resulting equations of motion duplicate the chiral ring. (For the moment, we have assumed no twisted masses are present; we will return to twisted masses at the end of this section.)

Now, to do meaningful computations, we must apply the D-term constraints to both $Y_{i}$ 's and $F_{i}$ 's. Applying the D-term constraints to the $F_{i}$ 's to write them in terms of $G_{A}$ 's (i.e. integrating out $\Sigma_{a}$ 's), and for simplicity suppressing the $\Upsilon_{a}$ constraints and setting the mass scale $\mu$ to unity, we have the expression

$$
W=-\sum_{A=1}^{N-k} G_{A}\left(\sum_{i} V_{i}^{A} \exp \left(-Y_{i}\right)+\sum_{i_{S}} D_{i_{S}}^{A} \exp \left(-Y_{i_{S}}\right)\right),
$$

where

$$
D_{i_{S}}^{A}=-\sum_{i, j} \sum_{a} V_{i}^{A} B_{i j} Q_{j}^{a}\left[\left(S^{-1}\right)^{T}\right]_{a i_{S}}
$$

Note when $B=0, D=0$, and the expression for $W$ above immediately reduces to its $(2,2)$ locus form. We will derive this expression for $D$ below.

In this language, the mirror map between $\mathrm{A} / 2$ - and $\mathrm{B} / 2$-model observables is defined by

$$
\sum_{a=1}^{k} \sum_{j=1}^{N}\left(\delta_{i j}+B_{i j}\right) Q_{j}^{a} \sigma_{a} \leftrightarrow \exp \left(-Y_{i}\right)=e^{\tilde{t}_{i}} \prod_{A=1}^{N-k} \exp \left(-V_{i}^{A} \theta_{A}\right)
$$

(Strictly speaking, we will see in examples that these equations define not only the operator mirror map plus some of the chiral ring relations.)

We can derive the operator mirror map above from the superpotential (3.1) by taking a derivative with respect to $F_{i}$, as before. Doing so, one finds

$$
Q_{i}^{a} \sigma_{a}-\exp \left(-Y_{i}\right)+\sum_{i_{S}, j, a} B_{i j} Q_{j}^{a}\left[\left(S^{-1}\right)^{T}\right]_{a i_{S}} \exp \left(-Y_{i_{S}}\right)=0
$$


For $i$ corresponding to columns of $S, B_{i j}=0$, and the expression above simplifies to

$$
S_{i_{S}}^{a} \sigma_{a}=\exp \left(-Y_{i_{S}}\right)
$$

Plugging this back in, we find

$$
Q_{i}^{a} \sigma_{a}-\exp \left(-Y_{i}\right)+\sum_{j, a} B_{i j} Q_{j}^{a} \sigma_{a}=0
$$

which is easily seen to be the operator mirror map (3.4).

We can apply the operator mirror map as follows. Recall that the constraints imply

$$
\sum_{i} Q_{i}^{a} Y_{i}=t_{a}
$$

hence

$$
\prod_{i} \exp \left(-Q_{i}^{a} Y_{i}\right)=\exp \left(-t_{a}\right)=q_{a},
$$

hence plugging in the proposed map (3.4) above, we have

$$
\prod_{i}\left(\sum_{a=1}^{k} \sum_{j=1}^{N}\left(\delta_{i j}+B_{i j}\right) Q_{j}^{a} \sigma_{a}\right)^{Q_{i}^{a}}=q_{a}
$$

which is the chiral ring relation in the A/2-twisted GLSM.

In passing, to make the method above work, it is important that the determinants appearing in quantum sheaf cohomology relations in e.g. [22-25] all factorize. In other words, recall that for a general tangent bundle deformation, the quantum sheaf cohomology ring relations take the form

$$
\prod_{\alpha}\left(\operatorname{det} M_{\alpha}\right)^{Q_{\alpha}^{a}}=q_{a}
$$

where $\alpha$ denotes a block of chiral fields with the same charges, and $M_{\alpha}$ encodes the $E$ 's, which will mix chiral superfields of the same charges. In order for the operator mirror map construction we have outlined above to work, it is necessary that each det $M_{\alpha}$ factorize into a product of factors, one for each matter chiral multiplet. This is ultimately the reason why in this paper we have chosen to focus on 'toric' deformations, in which each E's do not mix different matter chiral multiplets.

Now, in terms of the operator mirror map, let us derive the form of $D$ above in equation (3.3). The equations of motion from the superpotential (3.2) are given by

$$
\frac{\partial W}{\partial G_{A}}=\sum_{i} V_{i}^{A} \exp \left(-Y_{i}\right)+\sum_{i_{S}} D_{i_{S}}^{A} \exp \left(-Y_{i_{S}}\right)=0
$$

Now, we plug in the operator mirror map (3.4) above to get

$$
\sum_{i} V_{i}^{A}\left(\sum_{a} \sum_{j}\left(\delta_{i j}+B_{i j}\right) Q_{j}^{a} \sigma_{a}\right)+\sum_{i_{S}} D_{i_{S}}^{A}\left(\sum_{a} \sum_{j}\left(\delta_{i_{S} j}+B_{i_{S} j}\right) Q_{j}^{a} \sigma_{a}\right)=0 .
$$


Using the constraint

$$
\sum_{i} V_{i}^{A} Q_{i}^{a}=0
$$

the first $\delta_{i j}$ term vanishes, and furthermore, since the matrix $B$ is defined to vanish for indices from columns of $S$, we see that in the second term, $B_{i_{S} j}=0$, hence the equation above reduces to

$$
\sum_{i, j} \sum_{a} V_{i}^{A} B_{i j} Q_{j}^{a} \sigma_{a}+\sum_{i_{S}} \sum_{a} D_{i_{S}}^{A} S_{i_{S}}^{a} \sigma_{a}=0
$$

Since this should hold for all $\sigma_{a}$, we have that

$$
\sum_{i, j} V_{i}^{A} B_{i j} Q_{j}^{a}+\sum_{i_{S}} D_{i_{S}}^{A} S_{i_{S}}^{a}=0
$$

which can be solved to give expression (3.3) for $D$ above.

Thus, the expression for the superpotential (3.2) together with the operator mirror map (3.4) has equations of motion that duplicate the chiral ring.

In passing, one could also formally try to consider more general cases in which a submatrix $S \subset Q$ is not specified. One might then try to take the expression for the mirror superpotential to be of the form

$$
W=-\sum_{A=1}^{N-k} G_{A}\left(\sum_{i} V_{i}^{A} \exp \left(-Y_{i}\right)+\sum_{i} D_{i}^{A} \exp \left(-Y_{i}\right)\right),
$$

where now the $i$ index on $D$ is allowed to run over all chiral superfields, not just a subset. Following the methods above, one cannot uniquely solve for $D$ - one gets families of possible $D$ 's with undetermined coefficients, and we do not know how to argue that the correlation functions match for all such coefficients without restricting to subsets defined by choices $S \subset Q$.

Now, in principle, for $(0,2)$ theories defined by deformations of the $(2,2)$ locus, there is an analogue of twisted masses that one can add to the theory. In the $(2,2)$ case, twisted masses corresponded to replacing a vector multiplet by its vevs, so that only a residue of $\sigma$ survived. In $(0,2)$, by contrast, the vector multiplet does not contain $\sigma$, only the gauge field, gauginos, and auxiliary fields $D$, so we can no longer interpret the twisted mass in terms of replacing a vector multiplet with its vevs.

Instead, we can understand the analogue of a twisted mass in a $(0,2)$ theory corresponding to a deformation of the $(2,2)$ locus in terms of additions to $E_{i}=\bar{D}_{+} \Lambda^{i}$, for Fermi superfields $\Lambda^{i}$. In particular, the $(2,2)$ vector multiplet's $\sigma$ field enters GLSMs written in $(0,2)$ superfields as a factor in such $E$ 's, so twisted masses enter similarly, as terms of the form

$$
E_{i}=\tilde{m}_{i} \phi_{i}
$$

(where as usual we are admitting the possibilty of several toric symmetries, and simply giving each chiral superfield the possibility of its own twisted mass). Such terms are only possible if the $(0,2)$ superpotential has compatible $J$ 's, meaning that in order for 
supersymmetry to hold, one requires $E \cdot J=0$, as usual. This is a residue of the requirement in the $(2,2)$ theory that twisted masses arise from flavor symmetries.

We have already seen, in section 2.3 , how $(2,2)$ twisted masses can be represented in the mirror, described in $(0,2)$ superspace. To describe their combination with $E$ deformations is straightforward. Briefly, the $(0,2)$ mirror superpotential takes the form

$$
W=-\sum_{A=1}^{N-k} G_{A}\left(\sum_{i} V_{i}^{A} \exp \left(-Y_{i}\right)+\sum_{i_{S}} D_{i_{S}}^{A} \exp \left(-Y_{i_{S}}\right)\right)+\sum_{i=1}^{N} \sum_{A=1}^{N-k} G_{A} V_{i}^{A} \tilde{m}_{i}
$$

with $\left(D_{i_{S}}^{A}\right)$ defined as in (3.3), and the operator mirror map has the form

$$
\sum_{a=1}^{k} \sum_{j=1}^{N}\left(\delta_{i j}+B_{i j}\right) Q_{j}^{a} \sigma_{a}+\tilde{m}_{i} \leftrightarrow \exp \left(-Y_{i}\right)=e^{\tilde{t}_{i}} \prod_{A=1}^{N-k} \exp \left(-V_{i}^{A} \theta_{A}\right) .
$$

\section{Correlation functions}

In this section, we will argue formally that correlation functions in our proposed $(0,2)$ mirrors match those of the original theory. More precisely, we will compare closed-string correlation functions of A- or A/2-twisted GLSM $\sigma$ 's to corresponding correlation functions in B- or B/2-twisted Landau-Ginzburg models. (Often, the Landau-Ginzburg mirror will be an orbifold; we will only compare against untwisted sector correlation functions in such orbifolds.) Our computations will focus on genus zero computations, but in $(2,2)$ cases, in principle can be generalized to any genus. (See also [48] for related work.)

Before doing so, let us first outline in what sense correlation functions match. There are two possibilities:

- First, for special matrices $\left(V_{i}^{A}\right)$, we will argue that correlation functions match on the nose. In order for this to happen, we will need to require that the determinant of an invertible $k \times k$ submatrix of the charge matrix $Q$, match (up to sign) the determinant of a complementary ${ }^{5}(N-k) \times(N-k)$ submatrix of $\left(V_{i}^{A}\right)$.

- Alternatively, we can always formally rescale some of the $Y_{i} \mathrm{~s}$ (without introducing or removing orbifolds) to arrange for the determinants above to match, up to sign. In this case, the correlation functions of one theory are isomorphic to those of the other theory, but the numerical factors will not match on the nose. (Instead, the relations between numerical factors will be determined by the rescaling of the $Y_{i} \mathrm{~s}$.)

In either event, correlation functions will match.

\footnotetext{
'Complementary' in this case means that if the $k \times k$ matrix is defined by $i$ 's corresponding to certain chiral superfields, then those same chiral superfields cannot appear corresponding to any $i$ 's in the $(N-$ $k) \times(N-k)$ submatrix of $\left(V_{i}^{A}\right)$.
} 


\section{$4.1 \quad(2,2)$ supersymmetric cases}

We will first check that on the $(2,2)$ locus, the ansatz described above (i.e. the ansatz of [10]) generates matching correlation functions between the A-twisted GLSM and its B-twisted Landau-Ginzburg model mirror. (See also [49] for an analogous comparison of partition functions.)

First, let us consider correlation functions in an A-twisted GLSM. An exact expression is given for fully massive cases in e.g. [50, Eq. (4.77)]:

$$
\langle\mathcal{O}\rangle=\frac{(-)^{N_{c}}}{|W|} \frac{1}{(-2 \pi i)^{\mathrm{rk} G}} \sum_{\sigma_{P}} \mathcal{O} \frac{Z_{1-\text { loop }}}{H}
$$

where $G$ is the GLSM gauge group, $W$ its Weyl group, $N_{c}$ its rank,

$$
Z_{1-\text { loop }}=\prod_{i=1}^{N}\left(\sum_{a=1}^{k} Q_{i}^{a} \sigma_{a}\right)^{R\left(\Phi_{i}\right)-1}
$$

( $R\left(\Phi_{i}\right)$ the R-charge, which for simplicity we will assume to vanish,) $\sigma_{P}$ the vacua, and $H$ is the Hessian of the twisted one-loop effective action, meaning

$$
H=\operatorname{det}\left(\sum_{i} \frac{Q_{i}^{a} Q_{i}^{b}}{\sum_{c} Q_{i}^{c} \sigma_{c}}\right),
$$

using (up to factors) the twisted one-loop effective action in e.g. [51, Eq. (3.36)].

Now, up to irrelevant overall factors, there is an essentially identical expression for Landau-Ginzburg correlation functions [52], involving the Hessian of the superpotential rather than $H / Z_{1-\text { loop }}$ above. Therefore, to show that correlation functions match, we will argue that the $H / Z_{1-\text { loop }}$ above, computed for the A-twisted GLSM, matches the Hessian of superpotential derivatives for the mirror Landau-Ginzburg model.

First, since we are only interested in the determinant, we can rotate the charge matrix $\left(Q_{i}^{a}\right)$ by an element $U \in \mathrm{SL}(k, \mathbb{C})$ without changing the determinant:

$$
\operatorname{det}\left(\sum_{i} \frac{Q_{i}^{a} Q_{i}^{b}}{\sum_{c} Q_{i}^{c} \sigma_{c}}\right) \mapsto(\operatorname{det} U)^{2} \operatorname{det}\left(\sum_{i} \frac{Q_{i}^{a} Q_{i}^{b}}{\sum_{c} Q_{i}^{c} \sigma_{c}}\right)=\operatorname{det}\left(\sum_{i} \frac{Q_{i}^{a} Q_{i}^{b}}{\sum_{c} Q_{i}^{c} \sigma_{c}}\right) .
$$

Thus, it will be convenient to rotate the charge matrix to the form ${ }^{6}$

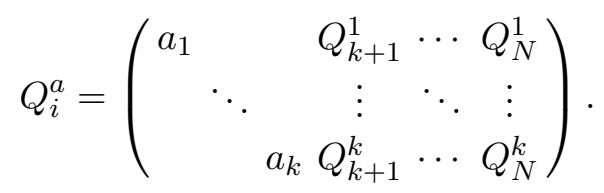

Note that for the charge matrix in this form,

$$
Z_{1 \text {-loop }}=\left(\prod_{i=1}^{k} a_{i} \sigma_{i}\right)^{-1}\left(\prod_{i=k+1}^{N}\left(\sum_{a=1}^{k} Q_{i}^{a} \sigma_{a}\right)\right)^{-1} .
$$

\footnotetext{
${ }^{6}$ As we are not conjugating the charge matrix, but rather multiplying on one side only, it should be possible to arrange for a $k \times k$ submatrix to be diagonal, not just in Jordan normal form.
} 
To put the charge matrix in this form, write

$$
Q=[S \mid *]=(\operatorname{det} S)^{1 / k}\left[S^{\prime} \mid *\right],
$$

where $S$ is $k \times k$. Then, multiply in $\left(S^{\prime}\right)^{-1}$, to get

$$
\left(S^{\prime}\right)^{-1} Q=(\operatorname{det} S)^{1 / k}[I \mid *],
$$

which is now diagonal.

It is straightforward to compute

$$
\begin{aligned}
H & =\operatorname{det}\left(\sum_{i} \frac{Q_{i}^{a} Q_{i}^{b}}{\sum_{c} Q_{i}^{c} \sigma_{c}}\right) \\
& =\operatorname{det}\left(\begin{array}{ccc}
\frac{a_{1}}{\sigma_{1}}+\frac{\left(Q_{k+1}^{1}\right)^{2}}{Q_{k+1}^{c} \sigma_{c}}+\ldots+\frac{\left(Q_{N}^{1}\right)^{2}}{Q_{N}^{c} \sigma_{c}} & \frac{Q_{k+1}^{1} Q_{k+1}^{2}}{Q_{k+1}^{c} \sigma_{c}}+\ldots+\frac{Q_{N}^{1} Q_{N}^{2}}{Q_{N}^{c} \sigma_{c}} & \ldots \\
\frac{Q_{k+1}^{2} Q_{k+1}^{1}}{Q_{k+1}^{c} \sigma_{c}}+\ldots \frac{Q_{N}^{2} Q_{N}^{1}}{Q_{N}^{c} \sigma_{c}} & \frac{a_{2}}{\sigma_{2}}+\frac{\left(Q_{k+1}^{2}\right)^{2}}{Q_{k+1}^{c} \sigma_{c}}+\ldots+\frac{\left(Q_{N}^{2}\right)^{2}}{Q_{N}^{c} \sigma_{c}} & \ldots \\
\vdots & \vdots & \ddots
\end{array}\right) .
\end{aligned}
$$

We define ${ }^{7} t_{i}=a_{i} / \sigma_{i}$ and $E_{i}^{a}=Q_{i}^{a} / \sqrt{\sum_{c} Q_{i}^{c} \sigma_{c}}$, then the matrix in the above determinant becomes

$$
\left(\begin{array}{ccc}
t_{1}+\left(E_{k+1}^{1}\right)^{2}+\ldots+\left(E_{N}^{1}\right)^{2} & E_{k+1}^{1} E_{k+1}^{2}+\ldots+E_{N}^{1} E_{N}^{2} & \cdots \\
E_{k+1}^{2} E_{k+1}^{1}+\ldots+E_{N}^{2} E_{N}^{1} & t_{2}+\left(E_{k+1}^{2}\right)^{2}+\ldots+\left(E_{N}^{2}\right)^{2} & \cdots \\
\vdots & \vdots & \ddots
\end{array}\right)
$$

When all the $t_{i}$ vanish, one can straightforwardly see that the matrix above is the product $\left(E^{T}\right)^{T} E^{T}$, for matrix $E$

$$
E=\left(\begin{array}{cccc}
E_{k+1}^{1} & E_{k+2}^{1} & \cdots & E_{N}^{1} \\
E_{k+1}^{2} & E_{k+2}^{2} & \cdots & E_{N}^{2} \\
\vdots & \vdots & \ddots & \vdots \\
E_{k+1}^{k} & \cdots & \cdots & E_{N}^{k}
\end{array}\right)
$$

Using standard results from linear algebra, the generalized characteristic polynomial of matrix (4.4), in terms of the variables $t_{i}$, is given by

$$
\sum_{m=0}^{k}\left(\sum_{a_{1}<\cdots<a_{m}} t_{a_{1}} \cdots t_{a_{m}} \operatorname{det}\left(M_{a_{1} \cdots a_{m}}\right)\right),
$$

where the matrix $M_{a_{1} \cdots a_{m}}$ denotes the submatrix of $M=\left(E^{T}\right)^{T} E^{T}$ by omitting rows $a_{1} \cdots a_{m}$ and columns $a_{1}^{\prime} \cdots a_{m}^{\prime}$ (i.e. a principal minor of $M$ of size $k-m$ ). (In our conventions, the determinant vanishes if $M$ has no entries.) Notice that $M=\left(E^{T}\right)^{T} E^{T}$, so the determinant can be written more simply as

$$
\operatorname{det} M+t_{1} \cdots t_{k}+\sum_{m=1}^{k-1}\left(\sum_{a_{1}<\cdots<a_{m}} t_{a_{1}} \cdots t_{a_{m}}\left(\sum_{i_{1}<\cdots<i_{N+m-2 k}}\left(\operatorname{det} E_{a_{1} \cdots a_{m}, i_{1} \cdots i_{N+m-2 k}}\right)^{2}\right)\right),
$$

\footnotetext{
${ }^{7}$ The reader should note that the $t_{i}$ in this section, defined above, is not related to $t$ 's used earlier to describe FI parameters.
} 
where det $E_{a_{1} \cdots a_{m}, i_{i} \cdots i_{N+m-2 k}}$ denotes the determinant of the submatrix of $E$ formed by omitting rows $a_{1} \cdots a_{m}$ and columns $i_{1} \cdots i_{N+m-2 k}$. (We formally require it to be zero when $N+m-2 k<0$.)

Finally, we divide by $Z_{1-\text { loop }}$ to get an expression for $H / Z_{1-\text { loop }}$ where $H$ is the Hes$\operatorname{sian}(4.1)$ :

$$
\frac{\operatorname{det} M+t_{1} \cdots t_{k}}{Z_{1-\text { loop }}}+\sum_{m=1}^{k-1}\left(\sum_{a_{1}<\cdots<a_{m}}\left(a_{a_{1}}\right)^{2} \cdots\left(a_{a_{m}}\right)^{2}\left(\prod_{i \notin\left\{a_{1}, \cdots, a_{m}\right\}}\left(\sum_{a=1}^{k} Q_{i}^{a} \sigma_{a}\right)\right) B_{a_{1} \cdots a_{m}}\right)
$$

for

$$
B_{a_{1} \cdots a_{m}}=\sum_{i_{1}<\cdots<i_{N+m-2 k}}\left(\operatorname{det} E_{a_{1} \cdots a_{m}, i_{1} \cdots i_{N+m-2 k}}\right)^{2}
$$

where $\operatorname{det} M$ vanishes for $N<2 k$. For later use, note that for $N \leq 2 k$ we can expand

$$
\frac{\operatorname{det} M}{Z_{1-\text { loop }}}=\left(\prod_{i=1}^{k} a_{i} \sigma_{i}\right)\left(\prod_{i \notin\left\{i_{1}, \cdots, i_{k}\right\}}\left(\sum_{c=1}^{k} Q_{k+i}^{c} \sigma_{c}\right)\left(A_{i_{1}, \cdots, i_{k}}\right)^{2}\right),
$$

and the terms for $1 \leq m \leq k-1$ are given by

$$
a_{a_{1}}^{2} \cdots a_{a_{m}}^{2}\left(\prod_{b \notin\left\{a_{1}, \cdots, a_{m}\right\}} a_{b} \sigma_{b}\right)\left(\prod_{i \notin\left\{i_{m+1}, \cdots, i_{k}\right\}}\left(\sum_{c=1}^{k} Q_{k+i}^{c} \sigma_{c}\right)\left(A_{i_{m+1}, \cdots, i_{k}}\right)^{2}\right)
$$

where $A_{i_{m+1}, \cdots, i_{k}}$ denotes the sum of determinants of all $(k-m) \times(k-m)$ submatrices of the charge matrix $\left(Q_{i}^{a}\right)$ for values of $i>k$.

Next, we need to compare the ratio $H / Z_{1-\text { loop }}$ above to the analogous Hessian arising in the mirror B-twisted Landau-Ginzburg model. Here, there is a nearly identical computation in which the Hessian we just computed is replaced with the determinant of second derivatives of the mirror superpotential (2.6):

$$
\begin{aligned}
\frac{\partial^{2} W}{\partial \theta_{A} \partial \theta_{B}} & =-\sum_{i=1}^{N}\left(e^{\tilde{t}_{i}}\left(\prod_{C=1}^{N-k} \exp \left(-V_{i}^{C} \theta_{C}\right)\right) V_{i}^{A} V_{i}^{B}\right) \\
& =-\sum_{i=1}^{N}\left(\left(\sum_{a=1}^{k} Q_{i}^{a} \sigma_{a}\right) V_{i}^{A} V_{i}^{B}\right)
\end{aligned}
$$

using the operator mirror map (2.7).

Thus, we need to compute

$$
\operatorname{det}\left(\sum_{i} V_{i}^{A} V_{i}^{B} \sum_{c} Q_{i}^{c} \sigma_{c}\right)
$$

and compare to the ratio $H / Z_{1-\text { loop }}$ from the A model that we computed previously. In principle, the argument here is very similar to the argument just given for the determinant 
defined by charge matrices. First, using the fact that $V$ has rank $N-k$, inside the determinant we can rotate $V$ to the more convenient form

$$
V_{i}^{A}=\left(\begin{array}{cccccc}
V_{1}^{1} & \cdots & V_{k}^{1} & \lambda^{1} & & \\
\vdots & \ddots & \vdots & & \ddots & \\
V_{1}^{k} & \cdots & V_{k}^{k} & & & \lambda^{(N-k)}
\end{array}\right)
$$

In fact, we can say more. Given that the $V$ matrix was originally defined to satisfy

$$
\sum_{i} Q_{i}^{a} V_{i}^{A}=0
$$

after the rotation above inside the determinant, the $V$ matrix should in fact have the form

$$
V_{i}^{A}=\left(\begin{array}{cccccc}
-\frac{\lambda^{1} Q_{k+1}^{1}}{a_{1}} & \cdots & -\frac{\lambda^{1} Q_{k+1}^{k}}{a_{k}} & \lambda^{1} & & \\
\vdots & \ddots & \vdots & & \ddots & \\
-\frac{\lambda^{(N-k)} Q_{N}^{1}}{a_{1}} & \cdots & -\frac{\lambda^{(N-k)} Q_{N}^{k}}{a_{k}} & & & \lambda^{(N-k)}
\end{array}\right)
$$

Then, using the more convenient form of $V$ above, we find that we can write the matrix

$$
\begin{aligned}
& \left(\sum_{i} V_{i}^{A} V_{i}^{B} \sum_{c} Q_{i}^{c} \sigma_{c}\right) \\
& \quad=\left(\begin{array}{ccc}
\left(\lambda^{1}\right)^{2}\left[\frac{\left(Q_{k+1}^{1}\right)^{2} \sigma_{1}}{a_{1}}+\cdots+\frac{\left(Q_{k+1}^{k}\right)^{2} \sigma_{k}}{a_{k}}+Q_{k+1}^{c} \sigma_{c}\right] & \lambda^{1} \lambda^{2}\left[\frac{Q_{k+1}^{1} Q_{k+2}^{1} \sigma_{1}}{a_{1}}+\cdots+\frac{Q_{k+1}^{k} Q_{k+2}^{k} \sigma_{k}}{a_{k}}\right] & \cdots \\
\lambda^{2} \lambda^{1}\left[\frac{Q_{k+1}^{1} Q_{k+2}^{1} \sigma_{1}}{a_{1}}+\cdots+\frac{Q_{k+1}^{k} Q_{k+2}^{k} \sigma_{k}}{a_{k}}\right] & \left(\lambda^{2}\right)^{2}\left[\frac{\left(Q_{k+2}^{1}\right)^{2} \sigma_{1}}{a_{1}}+\cdots+\frac{\left(Q_{k+2}^{k}\right)^{2} \sigma_{k}}{a_{k}}+Q_{k+2}^{c} \sigma_{c}\right] & \ldots \\
\vdots & \vdots & \ddots
\end{array}\right) .
\end{aligned}
$$

Similarly, we define $s_{i}=\left(\lambda^{i}\right)^{2} Q_{k+i}^{c} \sigma_{c}$ and $F_{i}^{a}=\lambda^{i} Q_{k+i}^{a} \sqrt{\sigma_{a} / a_{a}}$ (without summing over the index $a$ ). Then, the matrix above can be written as

$$
\left(\begin{array}{ccc}
s_{1}+\left(F_{1}^{1}\right)^{2}+\cdots+\left(F_{1}^{k}\right)^{2} & F_{1}^{1} F_{2}^{1}+\cdots+F_{1}^{k} F_{2}^{k} & \cdots \\
F_{2}^{1} F_{1}^{1}+\cdots+F_{2}^{k} F_{1}^{k} & s_{2}+\left(F_{2}^{1}\right)^{2}+\cdots+\left(F_{2}^{k}\right)^{2} & \cdots \\
\vdots & \vdots & \ddots
\end{array}\right)
$$

When all $s_{i}$ vanish, one can observe that the matrix is the product $F^{T} F$, for

$$
F=\left(\begin{array}{cccc}
F_{1}^{1} & F_{2}^{1} & \cdots & F_{N-k}^{1} \\
F_{1}^{2} & F_{2}^{2} & \cdots & F_{N-k}^{2} \\
\vdots & \vdots & \ddots & \vdots \\
F_{1}^{k} & F_{2}^{k} & \cdots & F_{N-k}^{k}
\end{array}\right)
$$

By using the same technique we can show that the determinant of (4.13) vanishes for $N>2 k$, and for $N \leq 2 k$ is

$$
\begin{aligned}
& \operatorname{det}\left(F^{T} F\right)+s_{1} \cdots s_{N-k} \\
& \quad+\sum_{n=1}^{N-k-1}\left(\sum_{i_{1}<\cdots<i_{n}}\left(s_{i_{1}} s_{i_{2}} \cdots s_{i_{n}}\right)\left(\sum_{a_{1}<\cdots<a_{2 k-N+n}}\left(\operatorname{det} F_{i_{1} \cdots i_{n}, a_{1} \cdots a_{2 k-N+n}}\right)^{2}\right)\right)
\end{aligned}
$$


For later use, note that

$$
\begin{aligned}
\operatorname{det} F^{T} F & =\sum_{a_{1}<\cdots<a_{2 k-N}}\left(\operatorname{det} F_{a_{1} \cdots a_{2 k-N}}\right)^{2}, \\
& =\left(\prod_{A=1}^{N-k}\left(\lambda^{A}\right)^{2}\right)\left(\prod_{b=1}^{k} \frac{\sigma_{b}}{a_{b}}\right)\left(\sum_{i_{1}<\cdots<i_{k}}\left(\sum_{a_{1}, \cdots, a_{k}} Q_{k+i_{1}}^{a_{1}} \cdots Q_{k+i_{k}}^{a_{k}} \epsilon_{a_{1} \cdots a_{k}}\right)^{2}\right),
\end{aligned}
$$

where $F_{a_{1} \cdots a_{2 k-N}}$ denotes the submatrix of $F_{i}^{a}$ formed by deleting columns $a_{1}$ through $a_{2 k-N}$.

Next, we plug

$$
s_{i_{j}}=\left(\lambda^{i_{j}}\right)^{2} Q_{k+i_{j}}^{c} \sigma_{c}
$$

into equation (4.16), and compare equation (4.8). First, note that we can expand

$$
\frac{t_{1} \cdots t_{k}}{Z_{1-\mathrm{loop}}}=\left(\prod_{i=1}^{k} a_{i}^{2}\right)\left(\prod_{j=1}^{N}\left(\sum_{a=1}^{k} Q_{j}^{a} \sigma_{a}\right)\right)
$$

which matches

$$
s_{1} \cdots s_{N-k}=\prod_{A=1}^{N-k}\left(\lambda^{A}\right)^{2}\left(\sum_{a=1}^{k} Q_{k+A^{A}}^{a} \sigma_{a}\right)
$$

so long as

$$
\prod_{A=1}^{N-k} \lambda^{A}= \pm \prod_{i=1}^{k} a_{i}
$$

Analogous results hold for other terms, as we now verify. First we consider the case $N \geq 2 k$. The term det $M / Z_{1-\text { loop }}$ in the previous determinant corresponds to the term $n=N-2 k$ in the expansion (4.16), which is given by

$$
\left(\prod_{A=1}^{N-k} \lambda^{A}\right)^{2} \prod_{a=1}^{k} \frac{\sigma_{a}}{a_{a}} \prod_{i \notin\left\{i_{1}, \cdots, i_{k}\right\}}\left(\sum_{c=1}^{k} Q_{k+i}^{c} \sigma_{c}\right)\left(A_{i_{1} \cdots i_{k}}\right)^{2}
$$

for $A_{i_{1} \cdots i_{k}}$ defined previously. It is easy to verify that this matches equation (4.9) for $\operatorname{det} M / Z_{1-\text { loop }}$ so long as condition (4.17) is satisfied, just as before. The remaining terms in expansion (4.16) for any given $n$ correspond to terms in (4.8) with $m$ related by $n=$ $N-2 k+m$, and have the form

$$
\left(\prod_{A=1}^{N-k} \lambda^{A}\right)^{2} \prod_{b \notin\left\{a_{1}, \cdots, a_{m}\right\}} \frac{\sigma_{b}}{a_{b}}\left(\prod_{i \notin\left\{i_{m+1}, \cdots, i_{k}\right\}}\left(\sum_{c=1}^{k} Q_{k+i}^{c} \sigma_{c}\right)\left(A_{i_{m+1} \cdots i_{k}}\right)^{2}\right),
$$

and it is easy to verify that this matches equation (4.10) so long as condition (4.17) is satisfied, just as before. The reader can now straightforwardly verify that analogous statements hold for the cases $k<N<2 k$, which exhausts all nontrivial possibilities.

Thus, we see that correlation functions will match so long as equation (4.17) holds. Furthermore, we can always arrange for equation (4.17) to hold. If it does not do so initially, 
then as discussed at the start of this section, we can perform field redefinitions and rescale $Y_{i}$ s to arrange for it to hold, at the cost of making the isomorphism between the correlation functions of either theory a shade more complicated. For example, the coefficient of

$$
\left(\sum_{c} Q_{k+1}^{c} \sigma_{c}\right) \cdots\left(\sum_{d} Q_{N}^{d} \sigma_{d}\right)
$$

in equation (4.8) is $\left(a_{1} a_{2} \cdots a_{k}\right)^{2}$, and the coefficient of the term of the same order in equation (4.16) is $\left(\lambda^{1} \lambda^{2} \cdots \lambda^{N-k}\right)^{2}$. We see that equation (4.17) is required for equality hold, and the choice of sign in equation (4.17) should not have any physical significance.

So far, we have worked at genus zero, but the same argument also implies that the same closed-string correlation functions match at arbitrary genus. At genus $g$, A-twisted GLSM correlation functions are computed in the same fashion albeit with a factor of $\left(H / Z_{1-\text { loop }}\right)^{g-1}$ (see e.g. [45, Section 4], [46, Section 5.1]), whereas B-twisted LandauGinzburg model correlation functions (in the untwisted sector) are computed with a factor of $\left(H^{\prime}\right)^{g-1}$ [52], for $H^{\prime}$ the determinant of second derivatives of the mirror superpotential. Demonstrating that $H / Z_{1-\text { loop }}=H^{\prime}$ therefore not only demonstrates that genus zero correlation functions match, but also higher-genus correlation functions. (For $(0,2)$ theories, by contrast, higher genus correlation functions are not yet understood, so there we will only be able to compare genus zero correlation functions.)

Essentially the same argument applies if one adds twisted masses to the theory. One simply makes the substitution

$$
\sum_{a} Q_{i}^{a} \sigma_{a} \rightarrow \sum_{a} Q_{i}^{a} \sigma_{a}+\widetilde{m}_{i}
$$

where $\widetilde{m}_{i}$ is the twisted mass. The details of the proof above are essentially unchanged. Also note that we are free to redefine $\sigma_{a}$ to $\sigma_{a}+c_{a}$, and we can use this to set the first $k$ twisted masses to zero. This leaves $N-k$ twisted masses, consistent with a global flavor symmetry $\mathrm{U}(1)^{N-k}$.

The arguments above hold so long as one can integrate out all of the matter Higgs fields, to obtain a pure Coulomb branch. In the $(2,2)$ theory one expects that one should be able to do this if one adds sufficient twisted masses (see e.g. [45, Section 2.3]). (In particular, adding twisted masses can act as a substitute for going far out along the Coulomb branch, which also makes the matter fields massive.)

\section{$4.2(0,2)$ supersymmetric cases}

We will now generalize the previous argument to $(0,2)$ cases.

Our argument here will be very similar to that given for $(2,2)$ cases. We will compare results for correlation functions in A/2-twisted GLSMs computed with supersymmetric localization to results for correlation functions computed in $\mathrm{B} / 2$-twisted $(0,2)$ LandauGinzburg models.

First, as before, applying supersymmetric localization to an A/2-twisted GLSM, there is an exact formula for (genus zero) $(0,2)$ correlation functions [25], which has more or 
less the same form as in the $(2,2)$ case, now involving a Hessian of derivatives of a twisted one-loop $(0,2)$ effective action [22], which takes the form

$$
H=\operatorname{det}\left(\sum_{i} \frac{\sum_{j} Q_{i}^{a} A_{i j} Q_{j}^{b}}{\sum_{m} A_{i m} Q_{m}^{c} \sigma_{c}}\right)
$$

where $A_{i j}=\delta_{i j}+B_{i j}$.

We assume without loss of generality that the invertible $S$ submatrix of the charge matrix corresponds to the first $k$ columns of $Q$. Then, one can show that the determinant (4.19) above is equal to

$$
\operatorname{det}\left(\begin{array}{ccc}
\frac{a_{1}}{\sigma_{1}}+\frac{Q_{k+1}^{1}\left(Q_{k+1}^{1}+\varepsilon_{k+1}^{1}\right)}{\left(Q_{k+1}^{a}+\varepsilon_{k+1}^{a}\right) \sigma_{a}}+\ldots+\frac{Q_{N}^{1}\left(Q_{N}^{1}+\varepsilon_{N}^{1}\right)}{\left(Q_{N}^{a}+\varepsilon_{N}^{a}\right) \sigma_{a}} & \frac{Q_{k+1}^{1}\left(Q_{k+1}^{2}+\varepsilon_{k+1}^{2}\right)}{\left(Q_{k+1}^{a}+\varepsilon_{k+1}^{a}\right) \sigma_{a}}+\ldots \frac{Q_{N}^{1}\left(Q_{N}^{2}+\varepsilon_{N}^{2}\right)}{\left(Q_{N}^{a}+\varepsilon_{N}^{a}\right) \sigma_{a}} & \ldots \\
\frac{Q_{k+1}^{2}\left(Q_{k+1}^{1}+\varepsilon_{k+1}^{2}\right)}{\left(Q_{k+1}^{a}+\varepsilon_{k+1}^{a}\right) \sigma_{a}}+\ldots \frac{Q_{N}^{2}\left(Q_{N}^{1}+\varepsilon_{N}^{1}\right)}{\left(Q_{N}^{a}+\varepsilon_{N}^{a}\right) \sigma_{a}} & \frac{a_{2}}{\sigma_{2}}+\frac{Q_{k+1}^{2}\left(Q_{k+1}^{2}+\varepsilon_{k+1}^{2}\right)}{\left(Q_{k+1}^{a}+\varepsilon_{k+1}^{a}\right) \sigma_{a}}+\ldots+\frac{Q_{N}^{2}\left(Q_{N}^{2} \varepsilon_{N}^{2}\right)}{\left(Q_{N}^{a}+\varepsilon_{N}^{a}\right) \sigma_{a}} \ldots \\
\vdots & \vdots & \ddots
\end{array}\right)
$$

where $\varepsilon_{i}^{a}=\sum_{j} B_{i j} Q_{j}^{a}$.

Now, in the $\mathrm{B} / 2$-twisted $(0,2)$ Landau-Ginzburg model, there is an analogous expression for correlation functions [17], involving the Hessian

$$
\operatorname{det} \frac{\partial^{2} W}{\partial G_{A} \partial \theta_{B}} .
$$

One can similarly show that the Hessian above is given by (using the $(0,2)$ operator mirror map (3.4))

$$
\operatorname{det}\left(\begin{array}{ccc}
\left(\lambda^{1}\right)^{2}\left[\sum_{b=1}^{k} \frac{Q_{k+1}^{b}\left(Q_{k+1}^{b}+\varepsilon_{k+1}^{b}\right) \sigma_{b}}{a_{b}}+S_{k+1}\right] & \lambda^{1} \lambda^{2}\left[\sum_{b=1}^{k} \frac{\left(Q_{k+1}^{b}+\varepsilon_{k+1}^{b}\right) Q_{k+2}^{b} \sigma_{b}}{a_{b}}\right] & \ldots \\
\lambda^{2} \lambda^{1}\left[\sum_{b=1}^{k} \frac{Q_{k+1}^{b}\left(Q_{k+2}^{b}+\varepsilon_{k+2}^{b}\right) \sigma_{b}}{a_{b}}\right] & \left(\lambda^{2}\right)^{2}\left[\sum_{b=1}^{k} \frac{Q_{k+2}^{b}\left(Q_{k+2}^{b}+\varepsilon_{k+2}^{b}\right) \sigma_{b}}{a_{b}}+S_{k+2}\right] & \ldots \\
\vdots & \vdots & \ddots
\end{array}\right)
$$

where $S_{k+i}=\sum_{a}\left(Q_{k+i}^{a}+\varepsilon_{k+i}^{a}\right) \sigma_{a}$.

Finally, following the same steps as for $(2,2)$, one can show that the ratio $H / Z_{1 \text {-loop }}$ appearing in the A/2-twisted GLSM matches the Hessian appearing in the B/2-twisted Landau-Ginzburg model,

$$
\operatorname{det}\left(\sum_{i} \frac{\sum_{j} Q_{i}^{a} A_{i j} Q_{j}^{b}}{\sum_{m} A_{i m} Q_{m}^{c} \sigma_{c}}\right)\left(\prod_{i=1}^{k} a_{i} \sigma_{i}\right)\left(\prod_{j=k+1}^{N}\left(Q_{j}^{a}+\epsilon_{j}^{a}\right) \sigma_{a}\right)=\operatorname{det} \frac{\partial^{2} W}{\partial G_{A} \partial \theta_{B}},
$$

so long as

$$
\prod_{i=1}^{N-k} \lambda^{i}= \pm \prod_{i=1}^{k} a_{i}
$$

(As before, if this does not hold, we can always perform field redefinitions to rescale some of the $Y_{i} \mathrm{~s}$ and corresponding Fermi fields $F_{i}$, at the cost of making the isomorphism between 
correlation functions of either theory slightly more complicated.) Thus, the A/2-twisted GLSM Hessian matches that arising in B/2-twisted Landau-Ginzburg model correlation functions [17]. Since correlation functions in the A/2-twisted GLSM and the B/2-twisted $(0,2)$ Landau-Ginzburg model have essentially the same form, albeit with potential different Hessians, and we have now demonstrated that the Hessians match, it follows that correlation functions match.

\section{Examples}

So far we have presented formal arguments for a $(0,2)$ mirror defined by a $(0,2)$ LandauGinzburg theory with the same chiral ring and correlation functions ${ }^{8}$ as the original A/2 theory. In this section we will verify that this proposal reproduces known results in specific examples.

To be specific, we will compare predictions to mirror proposals previously made in $[6,7]$. Those papers were originally written by guessing ansatzes for possible mirrors, constrained to match known results on the $(2,2)$ locus and to have the correct correlation functions and chiral ring relations. Here, we will see that the proposal we have presented correctly and systematically reproduces the results obtained by much more laborious methods in $[6,7]$. This will implicitly also provide tests that correlation functions and chiral rings do indeed match, as argued formally in the last section.

That said, the systematic proposal of this paper will only apply to special, 'toric' deformations, not all tangent bundle deformations, not even all tangent bundle deformations realizable by Euler-type sequences. Curiously, the terms in the mirrors described in $[6,7]$ that are not realized are nonlinear in the fields, suggesting that toric deformations are mirror to linear terms. We will not pursue this direction further in this paper, but mention it here for completeness.

\section{$5.1 \mathbb{P}^{1} \times \mathbb{P}^{1}$}

We begin by reviewing the now-nearly-canonical example of $\mathbb{P}^{1} \times \mathbb{P}^{1}$. The charge matrix of the GLSM for the chiral fields is of the form

$$
\left[\begin{array}{llll}
1 & 1 & 0 & 0 \\
0 & 0 & 1 & 1
\end{array}\right],
$$

and deformations of the tangent bundle are described mathematically as the cokernel of the short exact sequence

$$
0 \longrightarrow \mathcal{O}^{2} \stackrel{*}{\longrightarrow} \mathcal{O}(1,0)^{2} \oplus \mathcal{O}(0,1)^{2} \longrightarrow \mathcal{E} \longrightarrow 0,
$$

where the map $*$ is given by

$$
*=\left[\begin{array}{ll}
\tilde{A} x & \tilde{B} x \\
\tilde{C} y & \tilde{D} y
\end{array}\right],
$$

\footnotetext{
${ }^{8}$ Technically, untwisted sector correlation functions, if the mirror involves an orbifold.
} 
where $\tilde{A}, \tilde{B}, \tilde{C}, \tilde{D}$ are constant $2 \times 2$ matrices, and $x, y$ are two-component vectors of homogeneous coordinates on either $\mathbb{P}^{1}$ factor.

We have the same constraints on fields from $\mathrm{D}$ terms as on the $(2,2)$ locus, namely:

$$
Y_{1}+Y_{2}=t_{1}, \quad Y_{3}+Y_{4}=t_{2},
$$

where $Y_{1,2}$ are dual to the variables for one $\mathbb{P}^{1}$, and $Y_{3,4}$ are dual to the variables for the other.

Let us solve the constraints above by taking

$$
\tilde{t}_{1}=0, \quad \tilde{t}_{2}=t_{1}, \quad \tilde{t}_{3}=0, \quad \tilde{t}_{4}=t_{2},
$$

and

$$
V_{i}^{A}=\left[\begin{array}{cccc}
1 & -1 & 0 & 0 \\
0 & 0 & 1 & -1
\end{array}\right]
$$

so that

$$
\begin{aligned}
& Y_{1}=\theta, \quad Y_{2}=t_{1}-\theta, \quad G^{1}=F_{1}=-F_{2}, \\
& Y_{3}=\tilde{\theta}, \quad Y_{4}=t_{2}-\tilde{\theta}, \quad G^{2}=F_{3}=-F_{4} .
\end{aligned}
$$

\subsubsection{First choice of $S$}

We will first consider the case that the submatrix $S$ is given by the first and third columns of the charge matrix $Q$ above, so that $S$ is the identity matrix. Then, the allowed deformations are defined by

$$
A_{i j}=\left[\begin{array}{cccc}
1 & 0 & 0 & 0 \\
A_{21} & A_{22} & A_{23} & A_{24} \\
0 & 0 & 1 & 0 \\
A_{41} & A_{42} & A_{43} & A_{44}
\end{array}\right] .
$$

(Rows correspond to fixed $i$ index, and reflect the fact that for $i$ a row of $S$, values are fixed to those of the $(2,2)$ locus.) We can find the corresponding tangent bundle deformations by comparing the $E_{i}$ 's. In terms of the matrix above,

$$
\begin{aligned}
E_{1} & =\sum_{j, a} A_{1 j} Q_{j}^{a} \sigma_{a} \phi_{1}, \\
& =\sigma_{1} \phi_{1} \\
E_{2} & =\left(\left(A_{21}+A_{22}\right) \sigma_{1}+\left(A_{23}+A_{24}\right) \sigma_{2}\right) \phi_{2}, \\
E_{3} & =\sigma_{2} \phi_{3}, \\
E_{4} & \left.=\left(A_{41}+A_{42}\right) \sigma_{1}+\left(A_{43}+A_{44}\right) \sigma_{2}\right) \phi_{4},
\end{aligned}
$$

whereas in terms of $\tilde{A}, \tilde{B}, \tilde{C}, \tilde{D}$, we have

$$
\begin{aligned}
& E_{1}=\left(\tilde{A}_{11} \phi_{1}+\tilde{A}_{12} \phi_{2}\right) \sigma_{1}+\left(\tilde{B}_{11} \phi_{1}+\tilde{B}_{12} \phi_{2}\right) \sigma_{2}, \\
& E_{2}=\left(\tilde{A}_{21} \phi_{1}+\tilde{A}_{22} \phi_{2}\right) \sigma_{1}+\left(\tilde{B}_{21} \phi_{1}+\tilde{B}_{22} \phi_{2}\right) \sigma_{2}, \\
& E_{3}=\left(\tilde{C}_{11} \phi_{3}+\tilde{C}_{12} \phi_{4}\right) \sigma_{1}+\left(\tilde{D}_{11} \phi_{3}+\tilde{D}_{12} \phi_{4}\right) \sigma_{2}, \\
& E_{4}=\left(\tilde{C}_{21} \phi_{3}+\tilde{C}_{22} \phi_{4}\right) \sigma_{1}+\left(\tilde{D}_{21} \phi_{3}+\tilde{D}_{22} \phi_{4}\right) \sigma_{2},
\end{aligned}
$$


for $\phi_{1,2}$ corresponding to homogeneous coordinates on the first $\mathbb{P}^{1}$ factor and $\phi_{3,4}$ corresponding to homogeneous coordinates on the second. Comparing these two expressions, we find that in terms of the original $2 \times 2$ matrices, our ansatz is equivalent to the special case

$$
\begin{aligned}
& \tilde{A}=\left[\begin{array}{cc}
1 & 0 \\
0 & A_{21}+A_{22}
\end{array}\right], \quad \tilde{B}=\left[\begin{array}{cc}
0 & 0 \\
0 & A_{23}+A_{24}
\end{array}\right], \\
& \tilde{C}=\left[\begin{array}{cc}
0 & 0 \\
0 & A_{41}+A_{42}
\end{array}\right], \quad \tilde{D}=\left[\begin{array}{cc}
1 & 0 \\
0 & A_{43}+A_{44}
\end{array}\right] .
\end{aligned}
$$

From formula (3.3), we have that

$$
\left(D_{i_{S}}^{A}\right)=\left[\begin{array}{cc}
A_{21}+A_{22}-1 & A_{23}+A_{24} \\
A_{41}+A_{42} & A_{43}+A_{44}-1
\end{array}\right],
$$

where the $A$ index counts across rows, and the $i_{S}$ index counts across columns.

The $(0,2)$ superpotential of the proposed mirror is then given by $(3.2)$

$$
\begin{aligned}
W= & -G_{1}\left(e^{-Y_{1}}-e^{-Y_{2}}+\left(A_{21}+A_{22}-1\right) e^{-Y_{1}}+\left(A_{23}+A_{24}\right) e^{-Y_{3}}\right) \\
& -G_{2}\left(e^{-Y_{3}}-e^{-Y_{4}}+\left(A_{41}+A_{42}\right) e^{-Y_{1}}+\left(A_{43}+A_{44}-1\right) e^{-Y_{3}}\right),
\end{aligned}
$$

If we define $X_{i}=\exp \left(-Y_{i}\right)$, then the $(0,2)$ superpotential above can be written

$$
\begin{aligned}
W= & -G_{1}\left(\left(A_{21}+A_{22}\right) X_{1}-\frac{q_{1}}{X_{1}}+\left(A_{23}+A_{24}\right) X_{3}\right) \\
& -G_{2}\left(\left(A_{43}+A_{44}\right) X_{3}-\frac{q_{2}}{X_{3}}+\left(A_{41}+A_{42}\right) X_{1}\right) .
\end{aligned}
$$

The operator mirror map (3.4) in this case implies

$$
\begin{aligned}
& X_{1} \leftrightarrow \sigma_{1}, \\
& X_{3} \leftrightarrow \sigma_{2} .
\end{aligned}
$$

In fact, the operator mirror map statement earlier also implies

$$
\begin{aligned}
& X_{2}=\frac{q_{1}}{X_{1}} \leftrightarrow\left(A_{21}+A_{22}\right) \sigma_{1}+\left(A_{23}+A_{24}\right) \sigma_{2}, \\
& X_{4}=\frac{q_{2}}{X_{3}} \leftrightarrow\left(A_{41}+A_{42}\right) \sigma_{1}+\left(A_{43}+A_{44}\right) \sigma_{2} .
\end{aligned}
$$

These two statements are also redundant consequences of the equations of motion $\partial W / \partial G_{1,2}=0$, which are themselves consequences of the quantum sheaf cohomology relations, as we shall see momentarily.

Now, let us compare results from [6]. There, it was argued that the $(0,2)$ mirror could be represented as

$$
\begin{aligned}
W= & -G_{1}\left(a X_{1}^{\prime}+b \frac{\left(X_{2}^{\prime}\right)^{2}}{X_{1}^{\prime}}+\mu X_{2}^{\prime}-\frac{q_{1}}{X_{1}^{\prime}}\right) \\
& -G_{2}\left(d X_{2}^{\prime}+c \frac{\left(X_{1}^{\prime}\right)^{2}}{X_{2}^{\prime}}+\nu X_{1}^{\prime}-\frac{q_{2}}{X_{2}^{\prime}}\right),
\end{aligned}
$$


where

$$
\begin{gathered}
a=\operatorname{det} \tilde{A}, \quad b=\operatorname{det} \tilde{B}, \quad c=\operatorname{det} \tilde{C}, \quad d=\operatorname{det} \tilde{D}, \\
\mu=\operatorname{det}(\tilde{A}+\tilde{B})-\operatorname{det} \tilde{A}-\operatorname{det} \tilde{B} \\
\nu=\operatorname{det}(\tilde{C}+\tilde{D})-\operatorname{det} \tilde{C}-\operatorname{det} \tilde{D}
\end{gathered}
$$

and with operator mirror map

$$
X_{1}^{\prime} \leftrightarrow \sigma_{1}, \quad X_{2}^{\prime} \leftrightarrow \sigma_{2}
$$

In the present case,

$$
a=A_{21}+A_{22}, \quad b=0, \quad c=0, \quad d=A_{43}+A_{44}, \quad \mu=A_{23}+A_{24}, \quad \nu=A_{41}+A_{42},
$$

and it is easy to see that the mirror superpotential proposed here matches the specialization of that in [6], if we identify $X_{1}=X_{1}^{\prime}, X_{3}=X_{2}^{\prime}$.

In addition, the quantum sheaf cohomology ring in this model is [22-25]

$$
a \sigma_{1}^{2}+b \sigma_{2}+\mu \sigma_{1} \sigma_{2}=q_{1}, \quad c \sigma_{1}^{2}+d \sigma_{2}^{2}+\nu \sigma_{1} \sigma_{2}=q_{2},
$$

which in the present case matches the remaining mirror map equations (5.1), (5.2) above.

Altogether, we see that the mirror proposed here matches results in [6], giving us a consistency check not only of proposed mirror superpotentials but also implicitly correlation functions and chiral rings.

\subsubsection{Second choice of $S$}

Next, we consider the case that the submatrix $S$ is given by the first and fourth columns of the charge matrix $Q$, so that $S$ is the identity matrix, and the allowed deformations are

$$
\left(A_{i j}\right)=\left[\begin{array}{cccc}
1 & 0 & 0 & 0 \\
A_{21} & A_{22} & A_{23} & A_{24} \\
A_{31} & A_{32} & A_{33} & A_{34} \\
0 & 0 & 0 & 1
\end{array}\right] .
$$

(Rows correspond to fixed $i$ index, and reflect the fact that for $i$ a row of $S$, values are fixed to those of the $(2,2)$ locus.) In other words, in terms of the original $2 \times 2$ matrices, we restrict to the special case

$$
\begin{gathered}
\tilde{A}=\left[\begin{array}{cc}
1 & 0 \\
0 & A_{21}+A_{22}
\end{array}\right], \quad \tilde{B}=\left[\begin{array}{cc}
0 & 0 \\
0 & A_{23}+A_{24}
\end{array}\right], \\
\tilde{C}=\left[\begin{array}{cc}
A_{31}+A_{32} & 0 \\
0 & 0
\end{array}\right], \quad \tilde{D}=\left[\begin{array}{cc}
A_{33}+A_{34} & 0 \\
0 & 1
\end{array}\right] .
\end{gathered}
$$

From formula (3.3), we have that

$$
\left(D_{i_{S}}^{A}\right)=\left[\begin{array}{cc}
A_{21}+A_{22}-1 & A_{23}+A_{24} \\
-\left(A_{31}+A_{32}\right) & -\left(A_{33}+A_{34}-1\right)
\end{array}\right],
$$

where the $A$ index counts across rows, and the $i_{S}$ index counts across columns. 
The $(0,2)$ superpotential of the proposed mirror (3.2) is then given by

$$
\begin{aligned}
W= & -G_{1}\left(e^{-Y_{1}}-e^{-Y_{2}}+\left(A_{21}+A_{22}-1\right) e^{-Y_{1}}+\left(A_{23}+A_{24}\right) e^{-Y_{4}}\right) \\
& -G_{2}\left(e^{-Y_{3}}-e^{-Y_{4}}-\left(A_{31}+A_{32}\right) e^{-Y_{1}}-\left(A_{33}+A_{34}-1\right) e^{-Y_{4}}\right), \\
= & -G_{1}\left(\left(A_{21}+A_{22}\right) X_{1}-\frac{q_{1}}{X_{1}}+\left(A_{23}+A_{24}\right) X_{4}\right) \\
& -G_{2}\left(\frac{q_{2}}{X_{4}}-\left(A_{31}+A_{32}\right) X_{1}-\left(A_{33}+A_{34}\right) X_{4}\right),
\end{aligned}
$$

where $X_{i}=\exp \left(-Y_{i}\right)$ and with hindsight we have chosen to write the superpotential in terms of $X_{1}$ and $X_{4}$, to illuminate the relation to other mirrors.

Furthermore, the operator mirror map (3.4) implies

$$
\begin{aligned}
& X_{1} \leftrightarrow \sigma_{1}, \\
& X_{4} \leftrightarrow \sigma_{2}
\end{aligned}
$$

and also implies

$$
\begin{aligned}
& X_{2}=\frac{q_{1}}{X_{1}} \leftrightarrow\left(A_{21}+A_{22}\right) \sigma_{1}+\left(A_{23}+A_{24}\right) \sigma_{2}, \\
& X_{3}=\frac{q_{2}}{X_{4}} \leftrightarrow\left(A_{31}+A_{32}\right) \sigma_{1}+\left(A_{33}+A_{34}\right) \sigma_{2}
\end{aligned}
$$

The latter two relations are a redundant restatement of the chiral ring of the theory, which can be seen by comparing the equations of motion of the superpotential above, defined by $\partial W / \partial G_{A}=0$.

Now, in this case the mirror given in [6] is defined by

$$
a=A_{21}+A_{22}, \quad b=c=0, \quad d=A_{33}+A_{34}, \quad \mu=A_{23}+A_{24}, \quad \nu=A_{31}+A_{32},
$$

and so has the form

$$
\begin{aligned}
W= & -G_{1}\left(\left(A_{21}+A_{22}\right) X_{1}^{\prime}+\left(A_{23}+A_{24}\right) X_{2}^{\prime}-\frac{q_{1}}{X_{1}^{\prime}}\right) \\
& -G_{2}\left(\left(A_{33}+A_{34}\right) X_{2}^{\prime}+\left(A_{31}+A_{32}\right) X_{1}^{\prime}-\frac{q_{2}}{X_{2}^{\prime}}\right),
\end{aligned}
$$

with operator mirror map

$$
X_{1}^{\prime} \leftrightarrow \sigma_{1}, \quad X_{2}^{\prime} \leftrightarrow \sigma_{2}
$$

With the dictionary $X_{1}=X_{1}^{\prime}, X_{4}=X_{2}^{\prime}$ and a sign change on $G_{2}$, we see that the superpotential and operator mirror map predicted here match that in [6]. In passing, note that the fact that we reproduce the mirror of [6] implicitly gives an independent check of matching of correlation functions. 


\subsubsection{Comment on Adams-Basu-Sethi result}

Let us very briefly compare to the analysis of [3], which also examined $(0,2)$ mirrors to $\mathbb{P}^{1} \times \mathbb{P}^{1}$. They primarily considered tangent bundle deformations of the form [3, Eq. (255)]

$$
\tilde{A}=I=\tilde{D}, \quad \tilde{C}=0, \quad \tilde{B}=\left[\begin{array}{cc}
\epsilon_{1} & 0 \\
0 & \epsilon_{2}
\end{array}\right] .
$$

In principle, a deformation of this form is compatible with making a non-invertible choice of $S$, specifically the last two columns of the charge matrix, so that

$$
S=\left[\begin{array}{ll}
0 & 0 \\
1 & 1
\end{array}\right] .
$$

Since $S$ is not invertible, it is not possible to uniquely solve for $\left(D_{i_{S}}^{A}\right)$ i in our approach, and we find it intriguing that in their analysis, they also were not able to uniquely solve for the mirror superpotential without doing further worldsheet instanton computations to solve for the values of some otherwise-undetermined parameters.

\section{$5.2 \quad \mathbb{F}_{n}$}

Next we consider Hirzebruch surfaces $\mathbb{F}_{n}$. Now, for $n>1$, these are not Fano, but nevertheless one can write down a mirror for the GLSM (which for the non-Fano cases is more properly interpreted as a mirror to a different geometric phase, the UV phase, of the GLSM), as discussed in [7]. The charge matrix of the GLSM is

$$
\left[\begin{array}{llll}
1 & 1 & n & 0 \\
0 & 0 & 1 & 1
\end{array}\right]
$$

and deformations of the tangent bundle are described mathematically as the cokernel $\mathcal{E}$ of the short exact sequence

$$
0 \longrightarrow \mathcal{O}^{2} \stackrel{*}{\longrightarrow} \mathcal{O}(1,0)^{2} \oplus \mathcal{O}(n, 1) \oplus \mathcal{O}(0,1) \longrightarrow \mathcal{E} \longrightarrow 0
$$

where

$$
*=\left[\begin{array}{cc}
\tilde{A} x & \tilde{B} x \\
\gamma_{1} s & \gamma_{2} s \\
\alpha_{1} t & \alpha_{2} t
\end{array}\right] .
$$

In the expression above, $x$ is a two-component vector of homogeneous coordinates of charge $(1,0), s$ is a homogeneous coordinate of charge $(n, 1)$, and $t$ is a homogeneous coordinate of charge $(0,1), A, B$ are constant $2 \times 2$ matrices, and $\gamma_{1,2}, \alpha_{1,2}$ are constants. (In principle, nonlinear deformations are also possible, but as observed previously in e.g. [22-25], do not contribute to quantum sheaf cohomology or A/2-model correlation functions, so we omit nonlinear deformations.) The $(2,2)$ locus is given by the special case

$$
A=I, \quad B=0, \quad \gamma_{1}=n, \quad \gamma_{2}=1, \alpha_{1}=0, \quad \alpha_{2}=1 .
$$


We have the same constraints on fields from D terms as on the $(2,2)$ locus, namely

$$
Y_{1}+Y_{2}+n Y_{s}=t_{1}, \quad Y_{s}+Y_{t}=t_{2},
$$

where $Y_{1,2}$ are dual to the $x$ 's, $Y_{3}$ is dual to $s$, and $Y_{4}$ is dual to $t$. We can solve them by taking

$$
\begin{aligned}
& \tilde{t}_{1}=0, \quad \tilde{t}_{2}=t_{1}, \quad \tilde{t}_{s}=0, \quad \tilde{t}_{t}=t_{2}, \\
& \left(V_{i}^{A}\right)=\left[\begin{array}{cccc}
1 & -1 & 0 & 0 \\
0 & -n & 1 & -1
\end{array}\right] \text {, }
\end{aligned}
$$

so that

$$
\begin{array}{lll}
Y_{1}=\theta, & Y_{2}=t_{1}-\theta-n \tilde{\theta}, & G_{1}=F_{1}=-F_{2}-n G_{2}, \\
Y_{3}=\tilde{\theta}, & Y_{4}=t_{2}-\tilde{\theta}, & G_{2}=F_{3}=-F_{4} .
\end{array}
$$

\subsubsection{First choice of $S$}

We take the submatrix $S \subset Q$ to correspond to the first and third columns of the charge matrix $Q$, i.e.

$$
S=\left[\begin{array}{ll}
1 & n \\
0 & 1
\end{array}\right] \text {. }
$$

The allowed deformations are

$$
\left(A_{i j}\right)=\left[\begin{array}{cccc}
1 & 0 & 0 & 0 \\
A_{21} & A_{22} & A_{23} & A_{24} \\
0 & 0 & 1 & 0 \\
A_{41} & A_{42} & A_{43} & A_{44}
\end{array}\right] .
$$

To find the corresponding elements of $\tilde{A}, \tilde{B}, \gamma_{1,2}, \alpha_{1,2}$, we compare the E's. For the deformations defined by $A_{i j}$,

$$
\begin{aligned}
E_{1} & =\sum_{a} Q_{a}^{a} \sigma_{a} \phi_{1}=\sigma_{1} \phi_{1}, \\
E_{2} & =\sum_{j, a} A_{2 j} Q_{j}^{a} \sigma_{a} \phi_{2}, \\
& =\left(A_{21} \sigma_{1}+A_{22} \sigma_{1}+A_{23}\left(n \sigma_{1}+\sigma_{2}\right)+A_{24} \sigma_{2}\right) \phi_{2}, \\
E_{s} & =\left(n \sigma_{1}+\sigma_{2}\right) s, \\
E_{t} & =\left(A_{41} \sigma_{1}+A_{42} \sigma_{1}+A_{43}\left(n \sigma_{1}+\sigma_{2}\right)+A_{44} \sigma_{2}\right) t,
\end{aligned}
$$

whereas for the bundle deformation parameters,

$$
\begin{aligned}
& E_{1}=\left(\tilde{A}_{11} \phi_{1}+\tilde{A}_{12} \phi_{2}\right) \sigma_{1}+\left(\tilde{B}_{11} \phi_{1}+\tilde{B}_{12} \phi_{2}\right) \sigma_{2}, \\
& E_{2}=\left(\tilde{A}_{21} \phi_{1}+\tilde{A}_{22} \phi_{2}\right) \sigma_{1}+\left(\tilde{B}_{21} \phi_{1}+\tilde{B}_{22} \phi_{2}\right) \sigma_{2}, \\
& E_{s}=\gamma_{1} s \sigma_{1}+\gamma_{2} s \sigma_{2}, \\
& E_{t}=\alpha_{1} t \sigma_{1}+\alpha_{2} t \sigma_{2},
\end{aligned}
$$


from which we read off

$$
\begin{aligned}
& \tilde{A}=\left[\begin{array}{cc}
1 & 0 \\
0 & A_{21}+A_{22}+n A_{23}
\end{array}\right], \quad \tilde{B}=\left[\begin{array}{cc}
0 & 0 \\
0 & A_{23}+A_{24}
\end{array}\right], \\
& a=\operatorname{det} \tilde{A}=A_{21}+A_{22}+n A_{23}, \quad b=\operatorname{det} \tilde{B}=0, \quad \mu=A_{23}+A_{24}, \\
& \gamma_{1}=n, \quad \gamma_{2}=1, \quad \alpha_{1}=A_{41}+A_{42}+n A_{43}, \quad \alpha_{2}=A_{43}+A_{44} \text {. }
\end{aligned}
$$

Next, let us construct the mirror. From formula (3.3),

$$
\left(D_{i_{S}}^{A}\right)=\left[\begin{array}{cc}
A_{21}+A_{22}-n A_{24}-1 & A_{23}+A_{24} \\
n\left(A_{21}+A_{22}-n A_{24}\right)+\left(A_{41}+A_{42}-n A_{44}\right) & n\left(A_{23}+A_{24}\right)+\left(A_{43}+A_{44}\right)-1
\end{array}\right],
$$

From equation (3.2), the proposed mirror superpotential is then

$$
\begin{gathered}
W=-G_{1}\left(e^{-Y_{1}}-e^{-Y_{2}}+\left(A_{21}+A_{22}-n A_{24}-1\right) e^{-Y_{1}}+\left(A_{23}+A_{24}\right) e^{-Y_{3}}\right) \\
-G_{2}\left(-n e^{-Y_{2}}+e^{-Y_{3}}-e^{-Y_{4}}\right. \\
\quad+\left(n\left(A_{21}+A_{22}-n A_{24}\right)+\left(A_{41}+A_{42}-n A_{44}\right)\right) e^{-Y_{1}} \\
\left.\quad+\left(n\left(A_{23}+A_{24}\right)+\left(A_{43}+A_{44}\right)-1\right) e^{-Y_{3}}\right) \\
=-G_{1}\left(\left(A_{21}+A_{22}-n A_{24}\right) X_{1}-\frac{q_{1}}{X_{1} X_{3}^{n}}+\left(A_{23}+A_{24}\right) X_{3}\right) \\
-G_{2}\left(-n \frac{q_{1}}{X_{1} X_{3}^{n}}+\left(n\left(A_{23}+A_{24}\right)+\left(A_{43}+A_{44}\right)\right) X_{3}-\frac{q_{2}}{X_{3}}\right. \\
\left.\quad+\left(n\left(A_{21}+A_{22}-n A_{24}\right)+\left(A_{41}+A_{42}-n A_{44}\right)\right) X_{1}\right)
\end{gathered}
$$

where $X_{i}=\exp \left(-Y_{i}\right)$, with operator mirror map (3.4)

$$
\begin{aligned}
X_{1} & \leftrightarrow \sigma_{1}, \\
X_{2}=\frac{q_{1}}{X_{1} X_{3}^{n}} & \leftrightarrow\left(A_{21}+A_{22}\right) \sigma_{1}+A_{23}\left(n \sigma_{1}+\sigma_{2}\right)+A_{24} \sigma_{2}, \\
X_{3} & \leftrightarrow n \sigma_{1}+\sigma_{2}, \\
X_{4}=\frac{q_{2}}{X_{3}} & \leftrightarrow\left(A_{41}+A_{42}\right) \sigma_{1}+A_{43}\left(n \sigma_{1}+\sigma_{2}\right)+A_{44} \sigma_{2} .
\end{aligned}
$$

Note that the operator mirror map relations for $X_{2}, X_{4}$ are consequences of the equations of motion $\partial W / \partial G_{A}=0$.

For these deformations, the quantum sheaf cohomology ring is given by [22-25]

$$
Q_{(k)} Q_{(s)}^{n}=q_{1}, \quad Q_{(s)} Q_{(t)}=q_{2},
$$

where

$$
\begin{aligned}
& Q_{(k)}=\left(A_{21}+A_{22}+n A_{23}\right) \sigma_{1}^{2}+\left(A_{23}+A_{24}\right) \sigma_{1} \sigma_{2}, \\
& Q_{(s)}=n \sigma_{1}+\sigma_{2}, \quad Q_{(t)}=\left(A_{41}+A_{42}+n A_{43}\right) \sigma_{1}+\left(A_{43}+A_{44}\right) \sigma_{2} .
\end{aligned}
$$


It is straightforward to check that these relations are implied by the mirror map equations above.

A proposal was made in [7] for the Toda dual to a (GLSM for a) Hirzebruch surface. Briefly, the mirror superpotential had the form

$$
W=-G_{1} J_{1}-G_{2} J_{2}
$$

for $[7$, Section 4.2]

$$
\begin{aligned}
J_{1}= & a X_{1}+\mu_{A B}\left(X_{3}-n X_{1}\right)+b \frac{\left(X_{3}-n X_{1}\right)^{2}}{X_{1}}-q_{1} X_{1}^{-1}\left(\gamma_{1} X_{1}+\gamma_{2}\left(X_{3}-n X_{1}\right)\right)^{-n}, \\
J_{2}= & n\left(a X_{1}+\mu_{A B}\left(X_{3}-n X_{1}\right)+b \frac{\left(X_{3}-n X_{1}\right)^{2}}{X_{1}}\right)-\frac{n q_{1}}{X_{1}\left(\gamma_{1} X_{1}+\gamma_{2}\left(X_{3}-n X_{1}\right)\right)^{n}} \\
& -\frac{q_{2}}{X_{3}}+\frac{\left(\gamma_{1} X_{1}+\gamma_{2}\left(X_{3}-n X_{1}\right)\right)\left(\alpha_{1} X_{1}+\alpha_{2}\left(X_{3}-n X_{1}\right)\right)}{X_{3}},
\end{aligned}
$$

with operator mirror map

$$
X_{1} \leftrightarrow \sigma_{1}, \quad X_{3} \leftrightarrow n \sigma_{1}+\sigma_{2} .
$$

It is straightforward to check that the proposal of [7], reviewed above, specializes to our proposal here.

\subsubsection{Second choice of $S$}

Next, consider the case that the submatrix $S \subset Q$ is taken to correspond to the first and fourth columns of $Q$, i.e.

$$
S=\left[\begin{array}{ll}
1 & 0 \\
0 & 1
\end{array}\right]
$$

The allowed deformations are

$$
\left(A_{i j}\right)=\left[\begin{array}{cccc}
1 & 0 & 0 & 0 \\
A_{21} & A_{22} & A_{23} & A_{24} \\
A_{31} & A_{32} & A_{33} & A_{34} \\
0 & 0 & 0 & 1
\end{array}\right]
$$

Proceeding as before, the corresponding $\tilde{A}, \tilde{B}, \gamma_{1,2}, \alpha_{1,2}$ are given by

$$
\begin{aligned}
& \tilde{A}=\left[\begin{array}{cc}
1 & 0 \\
0 & A_{21}+A_{22}+n A_{23}
\end{array}\right], \quad \tilde{B}=\left[\begin{array}{cc}
0 & 0 \\
0 & A_{23}+A_{24}
\end{array}\right], \\
& a=\operatorname{det} \tilde{A}=A_{21}+A_{22}+n A_{23}, \quad b=\operatorname{det} \tilde{B}=0, \quad \mu=A_{23}+A_{24}, \\
& \gamma_{1}=A_{31}+A_{32}+n A_{33}, \quad \gamma_{2}=A_{33}+A_{34}, \\
& \alpha_{1}=0, \quad \alpha_{2}=1 .
\end{aligned}
$$

Next, let us construct the mirror. From formula (3.3),

$$
\left(D_{i_{S}}^{A}\right)=\left[\begin{array}{cc}
A_{21}+A_{22}+n A_{23}-1 & A_{23}+A_{24} \\
n\left(A_{21}+A_{22}+n A_{23}\right)-\left(A_{31}+A_{32}+n A_{33}\right) & n\left(A_{23}+A_{24}\right)-\left(A_{33}+A_{34}-1\right)
\end{array}\right] .
$$


From equation (3.2), the proposed mirror superpotential is then

$$
\begin{gathered}
W=-G_{1}\left(e^{-Y_{1}}-e^{-Y_{2}}+\left(A_{21}+A_{22}+n A_{23}-1\right) e^{-Y_{1}}+\left(A_{23}+A_{24}\right) e^{-Y_{4}}\right) \\
-G_{2}\left(-n e^{-Y_{2}}+e^{-Y_{3}}-e^{-Y_{4}}\right. \\
\quad+\left(n\left(A_{21}+A_{22}+n A_{23}\right)-\left(A_{31}+A_{32}+n A_{33}\right)\right) e^{-Y_{1}} \\
\left.\quad+\left(n\left(A_{23}+A_{24}\right)-\left(A_{33}+A_{34}-1\right)\right) e^{-Y_{4}}\right) \\
=-G_{1}\left(\left(A_{21}+A_{22}+n A_{23}\right) X_{1}-\frac{q_{1}}{q_{2}^{n}} \frac{X_{4}^{n}}{X_{1}}+\left(A_{23}+A_{24}\right) X_{4}\right) \\
-G_{2}\left(-n \frac{q_{1}}{q_{2}^{n}} \frac{X_{4}^{n}}{X_{1}}+\frac{q_{2}}{X_{4}}+\left(n\left(A_{23}+A_{24}\right)-\left(A_{33}+A_{34}\right)\right) X_{4}\right. \\
\left.\quad+\left(n\left(A_{21}+A_{22}+n A_{23}\right)-\left(A_{31}+A_{32}+n A_{33}\right)\right) X_{1}\right)
\end{gathered}
$$

where $X_{i}=\exp \left(-Y_{i}\right)$, with operator mirror map (3.4)

$$
\begin{aligned}
X_{1} & \leftrightarrow \sigma_{1}, \\
X_{2}=\frac{q_{1}}{q_{2}^{n}} \frac{X_{4}^{n}}{X_{1}} & \leftrightarrow\left(A_{21}+A_{22}\right) \sigma_{1}+A_{23}\left(n \sigma_{1}+\sigma_{2}\right)+A_{24} \sigma_{2}, \\
X_{3}=\frac{q_{2}}{X_{4}} & \leftrightarrow\left(A_{31}+A_{32}\right) \sigma_{1}+A_{33}\left(n \sigma_{1}+\sigma_{2}\right)+A_{34} \sigma_{2}, \\
X_{4} & \leftrightarrow \sigma_{2} .
\end{aligned}
$$

The operator mirror map relation for $X_{2}$ is a consequence of $\partial W / \partial G_{1}=0$, and the operator mirror map relation for $X_{3}$ is a consequence of that plus $\partial W / \partial G_{2}=0$.

A second proposal was made in [7] for the Toda dual to a (GLSM for a) Hirzebruch surface, in which the mirror superpotential had the form

$$
W=-G_{1} J_{1}-G_{2} J_{2},
$$

for $[7$, Section 4.2]

$$
\begin{aligned}
J_{1}= & \left(a X_{1}+\mu_{A B} X_{4}+b \frac{X_{4}^{2}}{X_{1}}\right)-\frac{q_{1}}{q_{2}^{n}} \frac{\left(\alpha_{1} X_{1}+\alpha_{2} X_{4}\right)^{n}}{X_{1}}, \\
J_{2}= & -n\left(a X_{1}+\mu_{A B} X_{4}+b \frac{X_{4}^{2}}{X_{1}}-\frac{q_{1}}{q_{2}^{n}} \frac{\left(\alpha_{1} X_{1}+\alpha_{2} X_{4}\right)^{n}}{X_{1}}\right) \\
& +\left(\alpha_{2} \gamma_{2} X_{4}+\gamma_{1} \alpha_{1} \frac{X_{1}^{2}}{X_{4}}+\left(\gamma_{1} \alpha_{2}+\gamma_{2} \alpha_{1}\right) X_{1}\right)-\frac{q_{2}}{X_{4}} .
\end{aligned}
$$

with operator mirror map

$$
X_{1} \leftrightarrow \sigma_{1}, \quad X_{4} \leftrightarrow \sigma_{2}
$$

It is straightforward to check that this proposal of [7] specializes to our proposal. 


\section{$5.3 d P_{2}$}

Let us now consider the del Pezzo surface $d P_{2}$, corresponding to $\mathbb{P}^{2}$ blown up at two points, which was also considered in [7]. The charge matrix of the GLSM for the chiral fields is of the form

$$
\left[\begin{array}{lllll}
1 & 1 & 1 & 0 & 0 \\
0 & 0 & 1 & 1 & 0 \\
1 & 0 & 0 & 0 & 1
\end{array}\right],
$$

and deformations of the tangent bundle are described mathematically as the cokernel $\mathcal{E}$ of the short exact sequence

$$
\begin{array}{r}
0 \longrightarrow \mathcal{O}^{3} \stackrel{*}{\rightarrow} \mathcal{O}(1,0,1) \oplus \mathcal{O}(1,0,0) \oplus \mathcal{O}(1,1,0) \oplus \mathcal{O}(0,1,0) \oplus \mathcal{O}(0,0,1) \\
\longrightarrow \mathcal{E} \longrightarrow 0
\end{array}
$$

where

$$
*=\left[\begin{array}{ccc}
\alpha_{1} \phi_{1} & \alpha_{2} \phi_{2} & \alpha_{3} \phi_{3} \\
\beta_{1} \phi_{1} & \beta_{2} \phi_{2} & \beta_{3} \phi_{3} \\
\gamma_{1} \phi_{1} & \gamma_{2} \phi_{2} & \gamma_{3} \phi_{3} \\
\delta_{1} \phi_{1} & \delta_{2} \phi_{2} & \delta_{3} \phi_{3} \\
\epsilon_{1} \phi_{1} & \epsilon_{2} \phi_{2} & \epsilon_{3} \phi_{3}
\end{array}\right]
$$

The $(2,2)$ locus is given by the special case

$$
\begin{array}{lll}
\alpha_{1}=1, & \alpha_{2}=0, & \alpha_{3}=1, \\
\beta_{1}=1, & \beta_{2}=\beta_{3}=0, & \\
\gamma_{1}=\gamma_{2}=1, & \gamma_{3}=0, & \delta_{3}=0, \\
\delta_{1}=0, & \delta_{2}=1, & \\
\epsilon_{1}=\epsilon_{2}=0, & \epsilon_{3}=1 . &
\end{array}
$$

We will take

$$
\left(V_{i}^{A}\right)=\left[\begin{array}{ccccc}
1 & -1 & 0 & 0 & -1 \\
0 & -1 & 1 & -1 & 0
\end{array}\right]
$$

\subsubsection{First choice of $S$}

For our first choice of $S$, we will take the first, third, and fifth columns of the charge matrix, so that

$$
S=\left[\begin{array}{lll}
1 & 1 & 0 \\
0 & 1 & 0 \\
1 & 0 & 1
\end{array}\right] .
$$

With this choice of $S$, the allowed deformations are

$$
\left(A_{i j}\right)=\left[\begin{array}{ccccc}
1 & 0 & 0 & 0 & 0 \\
A_{21} & A_{22} & A_{23} & A_{24} & A_{25} \\
0 & 0 & 1 & 0 & 0 \\
A_{41} & A_{42} & A_{43} & A_{44} & A_{45} \\
0 & 0 & 0 & 0 & 1
\end{array}\right] .
$$


We compute the corresponding bundle deformation parameters by comparing E's:

$$
\begin{aligned}
E_{1} & =\left(\alpha_{1} \sigma_{1}+\alpha_{2} \sigma_{2}+\alpha_{3} \sigma_{3}\right) \phi_{1}=\left(\sigma_{1}+\sigma_{3}\right) \phi_{1}, \\
E_{2} & =\left(\beta_{1} \sigma_{1}+\beta_{2} \sigma_{2}+\beta_{3} \sigma_{3}\right) \phi_{2}, \\
& =\left(A_{21}\left(\sigma_{1}+\sigma_{3}\right)+A_{22} \sigma_{1}+A_{23}\left(\sigma_{1}+\sigma_{2}\right)+A_{24} \sigma_{2}+A_{25} \sigma_{3}\right) \phi_{2}, \\
E_{3} & =\left(\gamma_{1} \sigma_{1}+\gamma_{2} \sigma_{2}+\gamma_{3} \sigma_{3}\right) \phi_{3}=\left(\sigma_{1}+\sigma_{2}\right) \phi_{3}, \\
E_{4} & =\left(\delta_{1} \sigma_{1}+\delta_{2} \sigma_{2}+\delta_{3} \sigma_{3}\right) \phi_{4}, \\
& =\left(A_{41}\left(\sigma_{1}+\sigma_{3}\right)+A_{42} \sigma_{1}+A_{43}\left(\sigma_{1}+\sigma_{2}\right)+A_{44} \sigma_{2}+A_{45} \sigma_{3}\right) \phi_{4}, \\
E_{5} & =\left(\epsilon_{1} \sigma_{1}+\epsilon_{2} \sigma_{2}+\epsilon_{3} \sigma_{3}\right) \phi_{5}=\sigma_{3} \phi_{5},
\end{aligned}
$$

from which we find

$$
\begin{aligned}
& \vec{\alpha}=(1,0,1), \quad \vec{\gamma}=(1,1,0), \quad \vec{\epsilon}=(0,0,1), \\
& \vec{\beta}=\left(A_{21}+A_{22}+A_{23}, A_{23}+A_{24}, A_{21}+A_{25}\right), \\
& \vec{\delta}=\left(A_{41}+A_{42}+A_{43}, A_{43}+A_{44}, A_{41}+A_{45}\right) .
\end{aligned}
$$

From formula (3.3), we have

$$
\left(D_{i_{S}}^{A}\right)=\left[\begin{array}{ccc}
A_{21}+A_{22}-1-A_{24} & A_{23}+A_{24} & -A_{22}+A_{24}+A_{25}+1 \\
A_{21}+A_{22}-A_{24}+ & A_{23}+A_{24}+A_{43}+A_{44}-1 & -A_{22}+A_{24}+A_{25} \\
A_{41}+A_{42}-A_{44} & -A_{42}+A_{44}+A_{45}
\end{array}\right] .
$$

The proposed mirror superpotential (3.2) is then

$$
\begin{gathered}
W=-G_{1}\left[\left(A_{21}+A_{22}-A_{24}\right) X_{1}-\frac{q_{1}}{X_{1} X_{3}}+\left(A_{23}+A_{24}\right) X_{3}+\left(-A_{22}+A_{24}+A_{25}\right) X_{5}\right] \\
-G_{2}\left[\left(A_{21}+A_{22}-A_{24}+A_{41}+A_{42}-A_{44}\right) X_{1}\right. \\
\quad-\frac{q_{1}}{X_{1} X_{3}}+\left(A_{23}+A_{24}+A_{43}+A_{44}\right) X_{3} \\
\left.\quad-\frac{q_{2}}{X_{3}}+\left(-A_{22}+A_{24}+A_{25}-A_{42}+A_{44}+A_{45}\right) X_{5}\right],
\end{gathered}
$$

with operator mirror map (3.4)

$$
\begin{aligned}
X_{1} & \leftrightarrow \sigma_{1}+\sigma_{3}, \\
X_{2}=\frac{q_{1}}{X_{1} X_{3}} & \leftrightarrow\left(A_{21}+A_{22}+A_{23}\right) \sigma_{1}+\left(A_{23}+A_{24}\right) \sigma_{2}+\left(A_{21}+A_{25}\right) \sigma_{3}, \\
X_{3} & \leftrightarrow \sigma_{1}+\sigma_{2}, \\
X_{4}=\frac{q_{2}}{X_{3}} & \leftrightarrow\left(A_{41}+A_{42}+A_{43}\right) \sigma_{1}+\left(A_{43}+A_{44}\right) \sigma_{2}+\left(A_{41}+A_{45}\right) \sigma_{3}, \\
X_{5}=\frac{q_{3}}{X_{1}} & \leftrightarrow \sigma_{3} .
\end{aligned}
$$

(It is straightforward to check that the nontrivial operator mirror map relations above are equivalent to the equations of motion derived from $\partial W / \partial G_{A}=0$.) 
Now, let us compare to the first $(0,2)$ mirror proposal for $d P_{2}$ in [7, Section 3.2.2]. In their notation

$$
\begin{aligned}
\alpha \cdot X & =X_{1}, \quad \gamma \cdot X=X_{3}, \quad \epsilon \cdot X=X_{5}, \\
\beta \cdot X & =\left(A_{21}+A_{22}-A_{24}\right) X_{1}+\left(A_{23}+A_{24}\right) X_{3}+\left(A_{24}+A_{25}-A_{22}\right) X_{5}, \\
\delta \cdot X & =\left(A_{41}+A_{42}-A_{44}\right) X_{1}+\left(A_{43}+A_{44}\right) X_{3}+\left(A_{44}+A_{45}-A_{22}\right) X_{5},
\end{aligned}
$$

and

$$
\begin{aligned}
J_{1} & =-\frac{q_{1}}{X_{1} X_{3}}+Z \frac{q_{3}}{X_{1} X_{5}}+X_{5}+\beta \cdot X, \\
J_{3} & =-\frac{q_{2}}{X_{3}}-\frac{q_{1}}{X_{1} X_{3}}+\beta \cdot X+\delta \cdot X, \\
J_{5} & =X_{5}+Z \frac{q_{3}}{X_{1} X_{5}}, \\
J_{Z} & =1-\frac{q_{3}}{X_{1} X_{5}} .
\end{aligned}
$$

Solving $J_{Z}=0$, we get $X_{5}=q_{3} / X_{1}$, and solving $J_{5}=0$, we get $Z=-X_{5}$. Plugging in, we are left with two independent $J$ 's:

$$
\begin{aligned}
J_{1}= & -\frac{q_{1}}{X_{1} X_{3}}+\left(A_{21}+A_{22}-A_{24}\right) X_{1}+\left(A_{23}+A_{24}\right) X_{3}+\left(A_{24}+A_{25}-A_{22}\right) \frac{q_{3}}{X_{1}}, \\
J_{3}= & -\frac{q_{2}}{X_{3}}-\frac{q_{1}}{X_{1} X_{3}}+\left(A_{21}+A_{22}-A_{24}+A_{41}+A_{42}-A_{44}\right) X_{1} \\
& +\left(A_{23}+A_{24}+A_{43}+A_{44}\right) X_{3}+\left(A_{24}+A_{25}-A_{22}+A_{44}+A_{45}-A_{42}\right) \frac{q_{3}}{X_{1}},
\end{aligned}
$$

which precisely matches the prediction of our proposal.

Finally, let us compare the quantum sheaf cohomology ring relations. There are three quantum sheaf cohomology ring relations, but only two relations appearing above in the operator mirror map and equations of motion. Specifically, in this case, the quantum sheaf cohomology ring relations [22-24] take the form

$$
\begin{aligned}
\left(\sigma_{1}+\sigma_{3}\right)\left(\sigma_{1}+\sigma_{2}\right) Q_{(2)} & =q_{1}, \\
\left(\sigma_{1}+\sigma_{2}\right) Q_{(4)} & =q_{2}, \\
\left(\sigma_{1}+\sigma_{3}\right) \sigma_{3} & =q_{3},
\end{aligned}
$$

where

$$
\begin{aligned}
& Q_{(2)}=\left(A_{21}+A_{22}+A_{23}\right) \sigma_{1}+\left(A_{23}+A_{24}\right) \sigma_{2}+\left(A_{21}+A_{25}\right) \sigma_{3}, \\
& Q_{(4)}=\left(A_{41}+A_{42}+A_{43}\right) \sigma_{1}+\left(A_{43}+A_{44}\right) \sigma_{2}+\left(A_{41}+A_{45}\right) \sigma_{3} .
\end{aligned}
$$

The first two quantum sheaf cohomology ring relations correspond to two of the operator mirror map statements. The third is realized on the mirror as the relation

$$
X_{1} X_{5}=q_{3}
$$

which is a consequence of the $\mathrm{D}$ terms, and so automatic. 


\subsubsection{Second choice of $S$}

For our second choice of $S$, we will take the second, fourth, and fifth columns of the charge matrix, so that $S$ is the identity. With that choice of $S$, the allowed deformations are

$$
\left(A_{i j}\right)=\left[\begin{array}{ccccc}
A_{11} & A_{12} & A_{13} & A_{14} & A_{15} \\
0 & 1 & 0 & 0 & 0 \\
A_{31} & A_{32} & A_{33} & A_{34} & A_{35} \\
0 & 0 & 0 & 1 & 0 \\
0 & 0 & 0 & 0 & 1
\end{array}\right] .
$$

To find the corresponding bundle deformation parameters, we compare the E's:

$$
\begin{aligned}
E_{1} & =\left(\alpha_{1} \sigma_{1}+\alpha_{2} \sigma_{2}+\alpha_{3} \sigma_{3}\right) \phi_{1}, \\
& =\left(A_{11}\left(\sigma_{1}+\sigma_{3}\right)+A_{12} \sigma_{1}+A_{13}\left(\sigma_{1}+\sigma_{2}\right)+A_{14} \sigma_{2}+A_{15} \sigma_{3}\right) \phi_{1}, \\
E_{2} & =\left(\beta_{1} \sigma_{1}+\beta_{2} \sigma_{2}+\beta_{3} \sigma_{3}\right) \phi_{2}=\sigma_{1} \phi_{2}, \\
E_{3} & =\left(\gamma_{1} \sigma_{1}+\gamma_{2} \sigma_{2}+\gamma_{3} \sigma_{3}\right) \phi_{3}, \\
& =\left(A_{31}\left(\sigma_{1}+\sigma_{3}\right)+A_{32} \sigma_{1}+A_{33}\left(\sigma_{1}+\sigma_{2}\right)+A_{34} \sigma_{2}+A_{35} \sigma_{3}\right) \phi_{3}, \\
E_{4} & =\left(\delta_{1} \sigma_{1}+\delta_{2} \sigma_{2}+\delta_{3} \sigma_{3}\right) \phi_{4}=\sigma_{2} \phi_{4}, \\
E_{5} & =\left(\epsilon_{1} \sigma_{1}+\epsilon_{2} \sigma_{2}+\epsilon_{3} \sigma_{3}\right) \phi_{5}=\sigma_{3} \phi_{5},
\end{aligned}
$$

from which we conclude

$$
\begin{aligned}
\vec{\alpha} & =\left(A_{11}+A_{12}+A_{13}, A_{13}+A_{14}, A_{11}+A_{15}\right), \\
\vec{\beta} & =(1,0,0), \quad \vec{\delta}=(0,1,0), \quad \vec{\epsilon}=(0,0,1), \\
\vec{\gamma} & =\left(A_{31}+A_{32}+A_{33}, A_{33}+A_{34}, A_{31}+A_{35}\right) .
\end{aligned}
$$

Next, we construct the mirror. From formula (3.3), we have

$$
\left(D_{i_{S}}^{A}\right)=-\left[\begin{array}{ccc}
A_{11}+A_{12}+A_{13}-1 & A_{13}+A_{14} & A_{11}+A_{15}-1 \\
A_{31}+A_{32}+A_{33}-1 & A_{33}+A_{34}-1 & A_{31}+A_{35}
\end{array}\right],
$$

then the proposed mirror superpotential (3.2) is

$$
\begin{aligned}
W= & -G_{1}\left(\frac{q_{1}}{q_{2}} \frac{X_{4}}{X_{2}}-\left(A_{11}+A_{12}+A_{13}\right) X_{2}-\left(A_{13}+A_{14}\right) X_{4}-\left(A_{11}+A_{15}\right) X_{5}\right) \\
& -G_{2}\left(-\left(A_{31}+A_{32}+A_{33}\right) X_{2}+\frac{q_{2}}{X_{4}}-\left(A_{33}+A_{34}\right) X_{4}-\left(A_{31}+A_{35}\right) X_{5}\right),
\end{aligned}
$$

where $X_{i}=\exp \left(-Y_{i}\right)$.

The operator mirror map (3.4) is then given by

$$
\begin{aligned}
X_{1}=\frac{q_{1}}{q_{2}} \frac{X_{4}}{X_{2}} & \leftrightarrow A_{11}\left(\sigma_{1}+\sigma_{3}\right)+A_{12} \sigma_{1}+A_{13}\left(\sigma_{1}+\sigma_{2}\right)+A_{14} \sigma_{2}+A_{15} \sigma_{3}, \\
X_{2} & \leftrightarrow \sigma_{1}, \\
X_{3}=\frac{q_{2}}{X_{4}} & \leftrightarrow A_{31}\left(\sigma_{1}+\sigma_{3}\right)+A_{32} \sigma_{1}+A_{33}\left(\sigma_{1}+\sigma_{2}\right)+A_{34} \sigma_{2}+A_{35} \sigma_{3}, \\
X_{4} & \leftrightarrow \sigma_{2}, \\
X_{5} & \leftrightarrow \sigma_{3} .
\end{aligned}
$$


From [22-24], the quantum sheaf cohomology relations in this model are given by

$$
\begin{aligned}
\sigma_{1} Q_{(1)} Q_{(3)} & =q_{1}, \\
\sigma_{2} Q_{(3)} & =q_{2}, \\
\sigma_{3} Q_{(1)} & =q_{3},
\end{aligned}
$$

where

$$
\begin{aligned}
& Q_{(1)}=\left(A_{11}+A_{12}+A_{13}\right) \sigma_{1}+\left(A_{13}+A_{14}\right) \sigma_{2}+\left(A_{11}+A_{15}\right) \sigma_{3}, \\
& Q_{(3)}=\left(A_{31}+A_{32}+A_{33}\right) \sigma_{1}+\left(A_{33}+A_{34}\right) \sigma_{2}+\left(A_{31}+A_{35}\right) \sigma_{3} .
\end{aligned}
$$

This first two quantum sheaf cohomology relations can be seen to correspond to both the operator mirror map relations and the equations of motion. The third relation (5.16) is effectively redundant on the mirror. To see this, note that it corresponds to the statement

$$
X_{5} Q_{(1)}=q_{3} .
$$

However, $X_{5}=q_{3} / X_{1}$ from the D-term relations, so this is equivalent to

$$
X_{1}=Q_{(1)}
$$

which is the first mirror-map relation.

\section{Hypersurfaces in toric varieties}

\subsection{General aspects}

An extension of ordinary mirror symmetry from toric varieties to hypersurfaces therein was discussed in [10] and further justified in [53]. Let us begin our discussion here by very briefly reviewing this for the special case of the $(2,2)$ quintic in $\mathbb{P}^{4}$. One begins with the Toda dual of Tot $\left(\mathcal{O}(-5) \rightarrow \mathbb{P}^{4}\right)$, the ambient GLSM for the quintic albeit with vanishing superpotential. The Toda dual is defined by a (twisted) superpotential of the form

$$
W=\sum_{i=1}^{5} \exp \left(-Y_{i}\right)+\exp \left(-Y_{p}\right),
$$

where the $\mathrm{D}$ term constraint requires

$$
-5 Y_{p}+\sum_{i=1}^{n} Y_{i}=t .
$$

The effect of dualizing the GLSM with a nonzero superpotential, according to $[10,53]$ is to change the fundamental fields from $Y_{i}$ to $X_{i} \equiv \exp \left(-Y_{i} / 5\right)$, which also introduces $\mathbb{Z}_{5}$ orbifolds. As a result, after eliminating $Y_{p}$ with the D term constraint and the change of variables, the mirror superpotential becomes $\left(\mathbb{Z}_{5}\right.$ orbifolds of $)$

$$
W=\sum_{i} X_{i}^{5}+q \prod_{i} X_{i}
$$


The fact that the B-twisted mirror sits at a Landau-Ginzburg point reflects the fact that the B model is independent of Kähler moduli, and so topological field theory computations can be computed at any point on the Kähler moduli space - the Landau-Ginzburg orbifold point in the moduli space of a hypersurface being a convenient example.

Before considering $(0,2)$ analogues, let us quickly outline a formal justification for the change of variables above, in TFT correlation functions. We claim that at the level of untwisted-sector correlation functions, it is formally equivalent to insertions needed to restrict to the hypersurface. The argument below omits questions of counting and degeneracy of vacua, as well as how the kinetic terms of the fields change, but is sufficiently tantalizing that we mention it here.

Begin with a $(2,2)$ supersymmetric GLSM for Tot $\left(\mathcal{O}(-5) \rightarrow \mathbb{P}^{4}\right)$, labelled the $V^{+}$ model in [51]. To compute a correlation function matching one on the hypersurface, in principle one should insert $(-)(-5 \sigma)^{2}$ (see e.g. [51, Eq. (5.8)]). Correlation functions in the untwisted sector of the mirror B-twisted Landau-Ginzburg model, corresponding to certain computations on the hypersurface, should then have the form

$$
\left\langle\mathcal{O}_{1} \cdots \mathcal{O}_{n}\right\rangle_{\text {quintic }}=\left\langle\mathcal{O}_{1} \cdots \mathcal{O}_{n}(-)(-5 \sigma)^{2}\right\rangle_{V^{+}} \propto \sum_{\text {vacua }} \frac{\mathcal{O}_{1} \cdots \mathcal{O}_{n} \exp \left(-2 Y_{p}\right)}{\operatorname{det}\left(\partial_{Y_{i}} \partial_{Y_{j}} W\right)}
$$

where we have used the mirror map

$$
\exp \left(-Y_{p}\right)=e^{-t / 5} \prod_{i} \exp \left(-Y_{i} / 5\right) \leftrightarrow-5 \sigma .
$$

Now, we change variables from $Y_{i}$ to $X_{i} \equiv \exp \left(-Y_{i} / 5\right)$. Formally, in the denominator,

$$
\begin{aligned}
\frac{\partial}{\partial Y_{i}} W & =\frac{\partial X_{j}}{\partial Y_{i}} \frac{\partial W}{\partial X_{j}}=-\frac{1}{5} X_{i} \frac{\partial W}{\partial X_{i}}, \\
\frac{\partial^{2} W}{\partial Y_{i} \partial Y_{j}} & =\left(-\frac{1}{5}\right)^{2} X_{i} X_{j} \frac{\partial^{2} W}{\partial X_{i} \partial X_{j}}(\text { no sum on } i, j),
\end{aligned}
$$

along the critical locus. Now, strictly speaking, after the change of variables, the critical locus becomes degenerate, the second derivative of the superpotential vanishes at the critical locus. One could attempt to solve this by turning on twisted masses, possible for certain special superpotentials, but as our aim here is a formal observation, we shall move on. The point we wish to make is that formally, if one plugs into the expression for correlation functions, then glossing over questions of number and degeneracy of vacua,

$$
\left\langle\mathcal{O}_{1} \cdots \mathcal{O}_{n}\right\rangle_{\text {quintic }}=\sum_{\text {vacua }} \frac{\mathcal{O}_{1} \cdots \mathcal{O}_{n} \exp \left(-2 Y_{p}\right)}{(-1 / 5)^{10}\left(\prod_{i} X_{i}\right)^{2} \operatorname{det}\left(\partial_{X_{i}} \partial_{X_{j}} W\right)},
$$

then along the vacua, the factor of $\exp \left(-2 Y_{p}\right)=e^{-2 t / n}\left(\prod_{i} X_{i}\right)^{2}$ largely cancels out the $\left(\prod_{i} X_{i}\right)^{2}$ in the denominator, leaving an expression which (setting aside questions of counting and degeneracy of vacua, as well as how the change of variables would operate on kinetic terms) formally duplicates the usual expression in the orbifold:

$$
\left\langle\mathcal{O}_{1} \cdots \mathcal{O}_{n}\right\rangle_{\text {quintic }} \propto \sum_{\text {vacua }} \frac{\mathcal{O}_{1} \cdots \mathcal{O}_{n}}{\operatorname{det}\left(\partial_{X_{i}} \partial_{X_{j}} W\right)} .
$$


Now, setting aside formal justifications, it is, of course, tempting to conjecture a $(0,2)$ analogue of the story above. The justification for the $(2,2)$ case in [53] was based on the claim in [54] that the A model on a hypersurface is equivalent to the A model on a supermanifold over the ambient space. Unfortunately, at present no $(0,2)$ analogue of [54] exists in the literature. Mathematically, the idea of inserting Chern classes to restrict to hypersurfaces is more properly understood in terms of insertions of Mathai-Quillen forms. A $(0,2)$ analogue of Mathai-Quillen forms has been sketched out (see [55] for definitions and conjectures), but one open question in that work is whether they have all the pertinent properties of ordinary Mathai-Quillen forms.

Another question concerns the appropriateness of getting a mirror at a LandauGinzburg orbifold point. In $(2,2)$ theories, for hypersurfaces, since the B model is independent of Kähler structures, we could evaluate a B-twisted mirror at any convenient point on the Kähler moduli space, and the Landau-Ginzburg orbifold point is a convenient such point. By contrast, $(0,2)$ theories are somewhat more complicated. It was argued in [22-25] that A/2-twisted GLSMs are independent of complex structure moduli and some bundle moduli $\left(J_{\mathrm{S}}\right)$, and B/2-twisted GLSMs are independent of Kähler moduli and other bundle moduli $(E \mathrm{~s})$, but this seems to be an accident of the GLSM's presentation of moduli, and we do not understand how to formulate analogous statements in IR nonlinear sigma models - or indeed if it is even possible to formulate such statements in IR theories. In any event, for our purposes, this will suffice to justify describing B/2-twisted mirrors at Landau-Ginzburg orbifold points.

With all that in mind, we will now describe some formal computations for $(0,2)$ theories, generated by proceeding along the same lines as $(2,2)$ theories, albeit with less justification.

Note that in $(0,2)$ theories, in addition to performing a variable change on $(0,2)$ chiral superfields, we must also perform a variable change on $(0,2)$ Fermi superfields. For example, suppose that in the mirror to the ambient GLSM, the fundamental fields are $\left(\theta_{A}, G_{A}\right)$, where $\theta_{A}$ s are $(0,2)$ chiral superfields and $G_{A}$ s are $(0,2)$ Fermi superfields, which on the $(2,2)$ locus together form a $(2,2)$ chiral multiplet. If we define

$$
X_{A}=\exp \left(-\theta_{A} / n\right)
$$

and take that to be a fundamental chiral superfield then to get a Landau-Ginzburg model that matches the $(2,2)$ mirror on the $(2,2)$ locus, we must also define new fundamental Fermi superfields $\Lambda_{A}$, as

$$
\Lambda_{A}=\frac{\partial X_{A}}{\partial \theta_{B}} G_{B} .
$$

In the example above,

$$
\frac{\partial X_{A}}{\partial \theta_{B}}=-\frac{1}{n} X_{A} \delta_{A}^{B},
$$

hence

$$
\Lambda_{A}=-\frac{1}{n} X_{A} G_{A}(\text { no sum on } A),
$$

and we should take $\Lambda_{A}$ to be the fundamental field in the mirror to the hypersurface, replacing $G_{A}$. 


\subsection{Example}

For an example, we will study the mirror to a $(0,2)$ GLSM describing a degree $(n, 0)$ hypersurface in $\mathbb{P}^{2} \times \mathbb{P}^{1}$, with a tangent bundle deformation. There are two dueling constraints that make finding interesting examples, somewhat nontrivial:

- Our E's, J's must satisfy $E \cdot J=0$ in order to preserve supersymmetry,

- Simultaneously, choices of $S \subset Q$ constrain the allowed deformations.

We will deal with these issues by considering a hypersurface in the first $\mathbb{P}^{2}$ factor, paired with a bundle deformation over the second $\mathbb{P}^{1}$ factor, so that the two constraints are fundamentally uncoupled from one another. We will 'follow our nose' and work out what naive expectations would predict for the $(0,2)$ mirror given the proposal of this paper; however, we have not checked any correlation functions or performed other independent tests to ensure that we have in fact produced the correct $(0,2)$ mirror.

Our GLSM will have six chiral superfields, which we label $\phi_{1, \cdots, 5}$ and $p$, with charge matrix

$$
Q=\left[\begin{array}{cccccc}
1 & 1 & 1 & 0 & 0 & -n \\
0 & 0 & 0 & 1 & 1 & 0
\end{array}\right]
$$

We interpret $\phi_{1-3}$ as related to homogeneous coordinates on first factor $\left(\mathbb{P}^{2}\right)$, and $\phi_{4-5}$ as related to homogeneous coordinates on the second factor $\left(\mathbb{P}^{1}\right)$.

Given the charge matrix $Q$ above, we will take

$$
\left(V_{i}^{A}\right)=\left[\begin{array}{cccccc}
n & 0 & 0 & 0 & 0 & 1 \\
0 & 1 & 0 & 0 & 0 & 1 / n \\
0 & 0 & 1 & 0 & 0 & 1 / n \\
0 & 0 & 0 & -1 & 1 & 0
\end{array}\right] .
$$

Now, since we are going to apply our mirror construction, we pick an invertible submatrix $S \subset Q$. To be specific, we will take $S$ to correspond to the fourth and sixth rows of $Q$, so that

$$
S=\left[\begin{array}{cc}
0 & -n \\
1 & 0
\end{array}\right],
$$

and we will consider deformations

$$
\left(A_{i j}\right)=\left[\begin{array}{cccccc}
1 & 0 & 0 & 0 & 0 & 0 \\
0 & 1 & 0 & 0 & 0 & 0 \\
0 & 0 & 1 & 0 & 0 & 0 \\
0 & 0 & 0 & 1 & 0 & 0 \\
A_{51} & A_{52} & A_{53} & A_{54} & A_{55} & A_{56} \\
0 & 0 & 0 & 0 & 0 & 1
\end{array}\right] .
$$

Now, the choice of $S$ above is compatible with a more complicated deformation matrix $\left(A_{i j}\right)$ - for example, the first, second, and third rows could have entries different from those of the identity matrix. However, to avoid running into difficulties with the constraint 
$E \cdot J=0$, for simplicity in this example we pick the $E$ 's to be trivial along directions with nonzero $J$ 's, and so we take the matrix to be of the more specialized form above.

With these choices, it is straightforward to compute

$$
\left(D_{i_{S}}^{A}\right)=\left[\begin{array}{cc}
0 & 0 \\
0 & 0 \\
0 & 0 \\
\left(A_{54}+A_{55}-1\right) / n-\left(A_{51}+A_{52}+A_{53}-n A_{56}\right)
\end{array}\right] .
$$

Thus, the $(0,2)$ mirror to the GLSM without superpotential is given by a LandauGinzburg model with superpotential

$$
\begin{gathered}
W=-G_{1}\left(n \exp \left(-Y_{1}\right)+\exp \left(-Y_{p}\right)\right)-G_{2}\left(\exp \left(-Y_{2}\right)+(1 / n) \exp \left(-Y_{p}\right)\right) \\
-G_{3}\left(\exp \left(-Y_{3}\right)+(1 / n) \exp \left(-Y_{p}\right)\right) \\
-G_{4}\left(-\exp \left(-Y_{4}\right)+\exp \left(-Y_{5}\right)+\frac{1}{n}\left(A_{54}+A_{55}-1\right) \exp \left(-Y_{4}\right)\right. \\
\left.-\left(A_{51}+A_{52}+A_{53}-n A_{56}\right) \exp \left(-Y_{p}\right)\right)
\end{gathered}
$$

subject to the usual D-term constraints, which allow us to rewrite $Y$ 's in terms of $\theta$ 's. If we pick

$$
\left(\tilde{t}_{i}\right)=\left(0,0,0, t_{2}, 0,-t_{1} / n\right)
$$

then

$$
\theta_{1}=Y_{1} / n, \quad \theta_{2}=Y_{2}, \quad \theta_{3}=Y_{3}, \quad \theta_{4}=Y_{5}
$$

and

$$
Y_{p}=\theta_{1}+\theta_{2} / n+\theta_{3} / n-t_{1} / n, \quad Y_{4}=-\theta_{4}+t_{2},
$$

so if we define

$$
Z_{1}=\exp \left(-\theta_{1}\right), \quad Z_{2,3}=\exp \left(-\theta_{2,3} / n\right), \quad Z_{4}=\exp \left(-\theta_{4}\right)
$$

the mirror superpotential above becomes

$$
\begin{aligned}
W= & -G_{1}\left(n Z_{1}^{n}+Z_{1} Z_{2} Z_{3} e^{-t_{1} / n}\right)-G_{2}\left(Z_{2}^{n}+\frac{1}{n} Z_{1} Z_{2} Z_{3} e^{-t_{1} / n}\right) \\
& -G_{3}\left(Z_{3}^{n}+\frac{1}{n} Z_{1} Z_{2} Z_{3} e^{-t_{1} / n}\right) \\
& -G_{4}\left(Z_{4}-\frac{q_{2}}{Z_{4}}+\frac{1}{n}\left(A_{54}+A_{55}-1\right) \frac{q_{2}}{Z_{4}}-\left(A_{51}+A_{52}+A_{53}-n A_{56}\right) Z_{1} Z_{2} Z_{3} e^{-t_{1} / n}\right),
\end{aligned}
$$

and the theory has a $\left(\mathbb{Z}_{n}\right)^{2}$ orbifold, acting on $Z_{2,3}$, in which the group action preserves the superpotential above. 
For completeness, the operator mirror map takes the following form:

$$
\begin{aligned}
\exp \left(-Y_{1}\right)=Z_{1}^{n} & \leftrightarrow \sigma_{1}, \\
\exp \left(-Y_{2}\right)=Z_{2}^{n} & \leftrightarrow \sigma_{1}, \\
\exp \left(-Y_{3}\right)=Z_{3}^{n} & \leftrightarrow \sigma_{1} \\
\exp \left(-Y_{4}\right)=\frac{q_{2}}{Z_{4}} & \leftrightarrow \sigma_{2} \\
\exp \left(-Y_{5}\right)=Z_{4} & \leftrightarrow\left(A_{51}+A_{52}+A_{53}-n A_{56}\right) \sigma_{1}+\left(A_{54}+A_{55}\right) \sigma_{2}, \\
\exp \left(-Y_{p}\right)=Z_{1} Z_{2} Z_{3} e^{-t_{1} / n} & \leftrightarrow-n \sigma_{1} .
\end{aligned}
$$

We could also add a twisted mass along the $p$ direction, for example. Doing so would add the following terms to the mirror Landau-Ginzburg model superpotential

$$
G_{A} V_{p}^{A} \tilde{m}_{p}=\tilde{m}_{p}\left(G_{1}+\frac{1}{n} G_{2}+\frac{1}{n} G_{3}\right)
$$

and also alter the operator mirror map, by modifying the map for $\exp \left(-Y_{p}\right)$ as

$$
\exp \left(-Y_{p}\right) \leftrightarrow-n \sigma_{1}+\tilde{m}_{p}
$$

Now, let us consider restricting to the hypersurface. This will involve changing the fundamental fields, from $\theta$ 's to $X$ 's and $G$ 's to $\Lambda$ 's.

First, let us consider changing variables amongst chiral superfields. We will take $Z_{i} \mathrm{~s}$ to be the fundamental variables. (Physically, this means changing e.g. kinetic terms, and so changing the physical theory, but here we will primarily focus on the superpotential.)

In addition, we must also change the Fermi fields, as mentioned previously. Following the pattern discussed previously, we define the new fundamental Fermi superfields

$$
\Lambda_{1}=-Z_{1} G_{1}, \quad \Lambda_{2,3}=-\frac{1}{n} Z_{2,3} G_{2,3}, \quad \Lambda_{4}=-Z_{4} G_{4} .
$$

Rewriting the mirror superpotential above in terms of the new fundamental fields $Z_{i}$, $\Lambda_{i}$, we find it takes the form

$$
\begin{aligned}
W= & \Lambda_{1}\left(n Z_{1}^{n-1}+Z_{2} Z_{3} e^{-t_{1} / n}\right)+\Lambda_{2}\left(n Z_{2}^{n-1}+Z_{1} Z_{3} e^{-t_{1} / n}\right) \\
& +\Lambda_{3}\left(n Z_{3}^{n-1}+Z_{1} Z_{2} e^{-t_{1} / n}\right) \\
& +\Lambda_{4}\left(1-\frac{q_{2}}{Z_{4}^{2}}+\frac{1}{n}\left(A_{54}+A_{55}-1\right) \frac{q_{2}}{Z_{4}^{2}}-\left(A_{51}+A_{52}+A_{53}-n A_{56}\right) \frac{Z_{1} Z_{2} Z_{3}}{Z_{4}} e^{-t_{1} / n}\right),
\end{aligned}
$$

again with a $\left(\mathbb{Z}_{n}\right)^{2}$ orbifold group action.

The reader should note that the first three terms in the superpotential above appear identical to those one would expect in a $(0,2)$ expansion of a $(2,2)$ superpotential of the form

$$
Z_{1}^{n}+Z_{2}^{n}+Z_{3}^{n}+Z_{1} Z_{2} Z_{3} e^{-t_{1} / n}
$$

exactly as one would expect for the mirror to a hypersurface of degree $n$ in $\mathbb{P}^{2}$. Similarly, the first two terms in the last line appear to be a $(0,2)$ expansion of the $(2,2)$ Toda dual to $\mathbb{P}^{1}$, defined by the superpotential

$$
Z_{4}+\frac{q_{2}}{Z_{4}}
$$

again exactly as one would expect. The remaining terms encode the bundle deformation. 


\subsection{Brief comment on the quintic}

Next, let us consider an example of a different character. Consider a GLSM for a quintic in $\mathbb{P}^{4}$. As is well-known (see e.g. [56, Section 15.6.3]), one can deform it to a $(0,2)$ theory. To see this, first rewrite the $(2,2)$ superpotential in $(0,2)$ language as

$$
W=\sum_{i} \Lambda^{i} J_{i}+\Lambda^{p} J_{p}
$$

for

$$
J_{i}=p \frac{\partial G}{\partial \phi_{i}}, \quad J_{p}=G
$$

for $G$ a quintic polynomial in the chiral superfields $\phi_{i}$. Then, one typically deforms off the $(2,2)$ locus by deforming

$$
J_{i}=p \frac{\partial G}{\partial \phi_{i}} \mapsto p \frac{\partial G}{\partial \phi_{i}}+G_{i}
$$

where the functions $G_{i}$ are constrained to obey

$$
\sum_{i} \phi_{i} G_{i}=0
$$

Now, let us consider this theory in the context of the mirror proposals of this paper. The A/2-twist only depends upon $E$ 's, not $J$ 's, so the deformation above is invisible in the twisted theory. The dualization procedure we have described does not seem to involve the $G_{i}$ 's above - we take a tangent bundle deformation on the ambient space and restrict to a hypersurface, but the $G_{i}$ 's cannot be understood as a tangent bundle deformation of the ambient space. As a result, using the methods here, the $G_{i}$ 's are effectively invisible. On the other hand, since the $\mathrm{A} / 2$ theory is independent of the $G_{i}$ 's, it is nevertheless a sensible mirror for the $\mathrm{A} / 2$ theory.

\section{Conclusions}

In this paper we have given a proposal for $(0,2)$ mirrors to toric Fano varieties with special tangent bundle deformations, subsets of toric deformations, and also described restrictions to hypersurfaces. Our methods do not apply to all tangent bundle deformations, only a subset of the toric deformations. We have given formal arguments that the resulting correlation functions always match, and also checked in examples that our methods reproduce previous results for $(0,2)$ mirrors produced by laboriously guessing and tuning ansatzes.

It would be interesting to generalize the results presented here to all tangent bundle deformations. On the one hand, for more general tangent bundle deformations, previous methods have generated nonlinear superpotential terms in the mirror, which we do not see. On the other hand, naive moduli counting arguments suggest that in many cases it may be possible to use field redefinitions to rewrite all the GLSM-realizable tangent bundle deformations in the form we use in this paper. 


\section{Acknowledgments}

We would like to thank L. Anderson, C. Closset, J. Gray, I. Melnikov, and R. Plesser for useful conversations. E.S. was partially supported by NSF grant PHY-1417410.

\section{A Brief notes on $(2,2)$ mirror ansatz}

In this appendix we will briefly outline how symmetries and the operator mirror map partially determine the exponential terms in the $(2,2)$ GLSM mirror superpotential. Suppose we have not derived the instanton-generated terms, and only have an ansatz for the mirror superpotential of the form

$$
W=\sum_{a=1}^{k} \Sigma_{a}\left(\sum_{i=1}^{N} Q_{i}^{a} Y_{i}-t_{a}\right)+g\left(Y_{i}\right),
$$

for some unknown function $g\left(Y_{i}\right)$. (Requiring R-charges match only fixes terms $\exp \left(-Y_{i}\right)$ up to an R-invariant function.) Instead of deriving $g$ from a direct instanton computation in the A-twisted theory, we outline here how the same result could be obtained using other properties of the theory.

Now, previously we derived the operator mirror map (2.7) from the form of the mirror superpotential, but one can outline an independent justification, and then use it to demonstrate the form of $g$. To see this, use the relation [10, Eq. (3.17)]

$$
Y+\bar{Y}=2 \bar{\Phi} e^{2 Q V} \Phi
$$

which implies component relations [10, Eqs. (3.20), (3.21)]

$$
\chi_{+}=2 \bar{\psi}_{+} \phi, \quad \bar{\chi}_{-}=-2 \phi^{\dagger} \psi_{-},
$$

for $\chi$ the superpartners of $Y$ and $\psi$ the superpartners of $\phi$. From [47, Eq. (2.19)], the equations of motion of $\bar{\sigma}_{a}$ (in the limit $e^{2} \rightarrow \infty$, so that the kinetic terms drop out) are

$$
\left(\sum_{i=1}^{N} Q_{i}^{a}\left|\phi_{i}\right|^{2}\right) \sigma_{a} \propto \sum_{i=1}^{N} \bar{\psi}_{+i} \psi_{-i} .
$$

If we add twisted masses so that only one $\phi$ field is light, then this becomes

$$
Q_{i}^{a} \sigma_{a}+\tilde{m}_{i} \propto \frac{\bar{\psi}_{+i} \psi_{-i}}{\left|\phi_{i}\right|^{2}} \propto \chi_{+i} \bar{\chi}_{-i}
$$

Now, the $\chi_{+} \bar{\chi}_{-}$could come from a $Y^{2}$, but that has the wrong $\mathrm{R}$ charge to make the expression sensible. However, $\exp \left(-Y_{i}\right)$ has the correct $\mathrm{R}$ charge and contains $Y^{2}$, so up to overall factors, which can be reabsorbed into field redefinitions, this suggests

$$
Q_{i}^{a} \sigma_{a}+\tilde{m}_{i}=\exp \left(-Y_{i}\right)
$$

which is the operator mirror map (2.7). (Granted, we are again using axial R-charges, but here since we know some components, there is less ambiguity.) 
Returning to the ansatz (A.1), we can now determine the function $g$. The equations of motion from the superpotential above imply

$$
\begin{aligned}
\frac{\partial W}{\partial Y_{i}} & =Q_{i}^{a} \sigma_{a}+\frac{\partial g}{\partial Y_{i}}, \\
& =0,
\end{aligned}
$$

and the operator mirror map implies

$$
Q_{i}^{a} \sigma_{a}+\tilde{m}_{a}=\exp \left(-Y_{i}\right),
$$

hence

$$
\frac{\partial g}{\partial Y_{i}}=-Q_{i}^{a} \sigma_{a}=\tilde{m}_{i}-\exp \left(-Y_{i}\right)
$$

hence

$$
g\left(Y_{i}\right)=\tilde{m}_{i} Y_{i}+\sum_{i} \exp \left(-Y_{i}\right),
$$

up to an irrelevant additive constant.

It is tempting to apply the same methods to $(0,2)$ theories. Unfortunately, the decreased symmetry leads to multiple possible potential $(0,2)$ mirror superpotentials, derived from applying the operator mirror map in different ways, which must be independently tested against chiral rings and correlation functions.

Open Access. This article is distributed under the terms of the Creative Commons Attribution License (CC-BY 4.0), which permits any use, distribution and reproduction in any medium, provided the original author(s) and source are credited.

\section{References}

[1] R. Blumenhagen, R. Schimmrigk and A. Wisskirchen, (0,2) mirror symmetry, Nucl. Phys. B 486 (1997) 598 [hep-th/9609167] [INSPIRE].

[2] R. Blumenhagen and S. Sethi, On orbifolds of (0,2) models, Nucl. Phys. B 491 (1997) 263 [hep-th/9611172] [INSPIRE].

[3] A. Adams, A. Basu and S. Sethi, (0,2) duality, Adv. Theor. Math. Phys. 7 (2003) 865 [hep-th/0309226] [INSPIRE].

[4] I.V. Melnikov and M.R. Plesser, A (0,2) mirror map, JHEP 02 (2011) 001 [arXiv: 1003.1303] [INSPIRE].

[5] I. Melnikov, S. Sethi and E. Sharpe, Recent developments in $(0,2)$ mirror symmetry, SIGMA 8 (2012) 068 [arXiv: 1209.1134] [INSPIRE].

[6] Z. Chen, E. Sharpe and R. Wu, Toda-like $(0,2)$ mirrors to products of projective spaces, JHEP 08 (2016) 093 [arXiv: 1603.09634] [INSPIRE].

[7] Z. Chen, J. Guo, E. Sharpe and R. Wu, More Toda-like (0,2) mirrors, JHEP 08 (2017) 079 [arXiv: 1705.08472] [INSPIRE].

[8] B.R. Greene and M.R. Plesser, Duality in Calabi-Yau moduli space, Nucl. Phys. B 338 (1990) 15 [inSPIRE]. 
[9] D.R. Morrison and M.R. Plesser, Towards mirror symmetry as duality for two-dimensional abelian gauge theories, Nucl. Phys. Proc. Suppl. 46 (1996) 177 [hep-th/9508107] [InSPIRE].

[10] K. Hori and C. Vafa, Mirror symmetry, hep-th/0002222 [INSPIRE].

[11] V.V. Batyrev, Dual polyhedra and mirror symmetry for Calabi-Yau hypersurfaces in toric varieties, J. Alg. Geom. 3 (1994) 493 [alg-geom/9310003] [INSPIRE].

[12] L. Borisov, Towards the mirror symmetry for Calabi-Yau complete intersections in toric Fano varieties, alg-geom/9310001.

[13] V. Batyrev and L. Borisov, Dual cones and mirror symmetry for generalized Calabi-Yau manifolds, alg-geom/9402002.

[14] S.H. Katz and E. Sharpe, Notes on certain $(0,2)$ correlation functions, Commun. Math. Phys. 262 (2006) 611 [hep-th/0406226] [INSPIRE].

[15] A. Adams, J. Distler and M. Ernebjerg, Topological heterotic rings, Adv. Theor. Math. Phys. 10 (2006) 657 [hep-th/0506263] [INSPIRE].

[16] E. Sharpe, Notes on certain other $(0,2)$ correlation functions, Adv. Theor. Math. Phys. 13 (2009) 33 [hep-th/0605005] [INSPIRE].

[17] I.V. Melnikov and S. Sethi, Half-twisted (0,2) Landau-Ginzburg models, JHEP 03 (2008) 040 [arXiv: 0712.1058] [INSPIRE].

[18] J. McOrist and I.V. Melnikov, Half-twisted correlators from the Coulomb branch, JHEP 04 (2008) 071 [arXiv: 0712.3272] [InSPIRE].

[19] M. Kreuzer, J. McOrist, I.V. Melnikov and M.R. Plesser, $(0,2)$ deformations of linear $\sigma$-models, JHEP 07 (2011) 044 [arXiv: 1001.2104] [INSPIRE].

[20] J. McOrist, The revival of $(0,2)$ linear $\sigma$-models, Int. J. Mod. Phys. A 26 (2011) 1 [arXiv: 1010.4667] [INSPIRE].

[21] J. Guffin, Quantum Sheaf cohomology, a precis, Mat. Contemp. 41 (2012) 17 [arXiv:1101.1305] [INSPIRE].

[22] J. McOrist and I.V. Melnikov, Summing the instantons in half-twisted linear $\sigma$-models, JHEP 02 (2009) 026 [arXiv:0810.0012] [INSPIRE].

[23] R. Donagi, J. Guffin, S. Katz and E. Sharpe, A mathematical theory of quantum sheaf cohomology, Asian J. Math. 18 (2014) 387 [arXiv:1110.3751] [INSPIRE].

[24] R. Donagi, J. Guffin, S. Katz and E. Sharpe, Physical aspects of quantum sheaf cohomology for deformations of tangent bundles of toric varieties, Adv. Theor. Math. Phys. 17 (2013) 1255 [arXiv: 1110.3752] [INSPIRE].

[25] C. Closset, W. Gu, B. Jia and E. Sharpe, Localization of twisted $\mathcal{N}=(0,2)$ gauged linear $\sigma$-models in two dimensions, JHEP 03 (2016) 070 [arXiv: 1512.08058] [INSPIRE].

[26] R. Donagi, Z. Lu and I.V. Melnikov, Global aspects of $(0,2)$ moduli space: toric varieties and tangent bundles, Commun. Math. Phys. 338 (2015) 1197 [arXiv:1409.4353] [INSPIRE].

[27] Z. Lu, A correlator formula for quantum sheaf cohomology, arXiv:1511.09158 [INSPIRE].

[28] J. Guo, Z. Lu and E. Sharpe, Quantum sheaf cohomology on Grassmannians, Commun. Math. Phys. 352 (2017) 135 [arXiv:1512.08586] [INSPIRE].

[29] J. Guo, Z. Lu and E. Sharpe, Classical sheaf cohomology rings on Grassmannians, J. Algebra 486 (2017) 246 [arXiv: 1605.01410] [INSPIRE]. 
[30] P. Candelas et al., A pair of Calabi-Yau manifolds as an exactly soluble superconformal theory, Nucl. Phys. B359 (1991) 21 [AMS/IP Stud. Adv. Math. 9 (1998) 31].

[31] V. Pestun, Localization of gauge theory on a four-sphere and supersymmetric Wilson loops, Commun. Math. Phys. 313 (2012) 71 [arXiv:0712.2824] [InSPIRE].

[32] F. Benini and S. Cremonesi, Partition functions of $\mathcal{N}=(2,2)$ gauge theories on $S^{2}$ and vortices, Commun. Math. Phys. 334 (2015) 1483 [arXiv:1206.2356] [InSPIRE].

[33] N. Doroud, J. Gomis, B. Le Floch and S. Lee, Exact results in D $=2$ supersymmetric gauge theories, JHEP 05 (2013) 093 [arXiv:1206.2606] [inSPIRE].

[34] S. Schäfer-Nameki and T. Weigand, F-theory and 2d (0,2) theories, JHEP 05 (2016) 059 [arXiv: 1601.02015] [INSPIRE].

[35] S. Franco, S. Lee and R.-K. Seong, Brane brick models and 2d $(0,2)$ triality, JHEP 05 (2016) 020 [arXiv: 1602.01834] [INSPIRE].

[36] F. Apruzzi, F. Hassler, J.J. Heckman and I.V. Melnikov, UV completions for non-critical strings, JHEP 07 (2016) 045 [arXiv: 1602.04221] [INSPIRE].

[37] A. Gadde and P. Putrov, Exact solutions of $(0,2)$ Landau-Ginzburg models, arXiv: 1608.07753 [INSPIRE].

[38] S. Franco, S. Lee, R.-K. Seong and C. Vafa, Brane brick models in the mirror, JHEP 02 (2017) 106 [arXiv: 1609.01723] [InSPIRE].

[39] S. Franco, S. Lee and R.-K. Seong, Orbifold reduction and $2 d(0,2)$ gauge theories, JHEP 03 (2017) 016 [arXiv: 1609.07144] [INSPIRE].

[40] F. Apruzzi, F. Hassler, J.J. Heckman and I.V. Melnikov, From 6D SCFTs to dynamic GLSMs, Phys. Rev. D 96 (2017) 066015 [arXiv:1610.00718] [InSPIRE].

[41] C. Lawrie, S. Schäfer-Nameki and T. Weigand, The gravitational sector of $2 d(0,2)$ F-theory vacua, JHEP 05 (2017) 103 [arXiv:1612.06393] [INSPIRE].

[42] S. Franco, D. Ghim, S. Lee and R.-K. Seong, Elliptic genera of $2 d(0,2)$ gauge theories from brane brick models, JHEP 06 (2017) 068 [arXiv: 1702.02948] [INSPIRE].

[43] M. Dedushenko, S. Gukov and P. Putrov, Vertex algebras and 4-manifold invariants, arXiv: 1705.01645 [INSPIRE].

[44] R. Tatar, From $N=2$ supersymmetry in four dimensions to $(0,2)$ supersymmetry in two dimensions, Phys. Rev. D 96 (2017) 066022 [arXiv:1706.04925] [INSPIRE].

[45] N.A. Nekrasov and S.L. Shatashvili, Bethe/Gauge correspondence on curved spaces, JHEP 01 (2015) 100 [arXiv: 1405.6046] [INSPIRE].

[46] F. Benini and A. Zaffaroni, A topologically twisted index for three-dimensional supersymmetric theories, JHEP 07 (2015) 127 [arXiv:1504.03698] [INSPIRE].

[47] E. Witten, Phases of $N=2$ theories in two-dimensions, Nucl. Phys. B 403 (1993) 159 [hep-th/9301042] [INSPIRE].

[48] C. Closset, N. Mekareeya and D.S. Park, A-twisted correlators and Hori dualities, JHEP 08 (2017) 101 [arXiv: 1705.04137] [INSPIRE].

[49] J. Gomis and S. Lee, Exact Kähler potential from gauge theory and mirror symmetry, JHEP 04 (2013) 019 [arXiv: 1210.6022] [INSPIRE]. 
[50] C. Closset, S. Cremonesi and D.S. Park, The equivariant A-twist and gauged linear $\sigma$-models on the two-sphere, JHEP 06 (2015) 076 [arXiv:1504.06308] [INSPIRE].

[51] D.R. Morrison and M.R. Plesser, Summing the instantons: quantum cohomology and mirror symmetry in toric varieties, Nucl. Phys. B 440 (1995) 279 [hep-th/9412236] [INSPIRE].

[52] C. Vafa, Topological Landau-Ginzburg models, Mod. Phys. Lett. A 6 (1991) 337 [InSPIRE].

[53] M. Aganagic and C. Vafa, Mirror symmetry and supermanifolds, Adv. Theor. Math. Phys. 8 (2004) 939 [hep-th/0403192] [inSPIRE].

[54] A.S. Schwarz, $\sigma$-models having supermanifolds as target spaces, Lett. Math. Phys. 38 (1996) 91 [hep-th/9506070] [INSPIRE].

[55] R.S. Garavuso and E. Sharpe, Analogues of Mathai-Quillen forms in sheaf cohomology and applications to topological field theory, J. Geom. Phys. 92 (2015) 1 [arXiv:1310.5754] [INSPIRE].

[56] M.B. Green, J.H. Schwarz and E. Witten, Superstring theory. Volume 2: loop amplitudes, anomalies and phenomenology, Cambridge Monographs On Mathematical Physics, Cambridge University Press, Cambridge U.K. (1987). 